\title{
Actividad hidráulica de un cemento belítico obtenido a partir de cenizas volantes tipo C: influencia del aditivo y tipo de curado
}

\author{
Hydraulic activity of belite cement from class C coal fly ash. \\ Effect of curing and admixtures
}

\author{
$\underline{\text { S. Goñi }}^{(*)}$ y A. Guerrero(*)
}

Recepción/Received: 21-IX-05

Aceptación/Accepted: 21-XII-05

\section{RESUMEN}

En este trabajo se discute la influencia del tipo de curado y de un aditivo reductor de la demanda de agua en la actividad hidráulica de un cemento belítico de cenizas volantes de alto contenido en cal denominado (CBCV-2A). Este cemento ha sido sintetizado por una ruta húmeda hidrotermal con posterior calcinación, empleando ceniza volante de alto contenido en cal (ASTM tipo C) como materia prima. La actividad hidráulica se ha estudiado en la pasta de cemento, durante un periodo de 180 días, por medio del contenido de agua combinada, determinada por análisis termogravimétrico, y el grado de hidratación por difracción de rayos X (DRX). La resistencia mecánica y la porosidad total y distribución de tamaño de poro se han estudiado en probetas equivalentes de mortero.

Palabras clave: cemento belítico, cenizas volantes, ruta hidrotermal, actividad hidráulica, aditivo.

\section{SUMMARY}

The effect of curing method and a water-reducing additive on the hydraulic activity of high lime content (ASTM type C) fly ash belite cement (FABC-2-W) is reported. $A$ class $C$ fly ash was subjected to hydrothermal treatment and subsequent calcination to synthesize FABC. Hydraulic activity was evaluated in the cement paste over 180 days from the physically bound water content as determined by thermogravimetric analysis and the degree of hydration, in turn found with X-ray diffraction (XRD) analysis. Mechanical strength, porosity and pore size distribution were also studied in equivalent mortar samples.

Keywords: belite cement, fly ash, hydrothermal calcination treatment, hydraulic activity, admixture.

\section{INTRODUCCIÓN}

En la Unión Europea de los 25 (UE 25) se producen cada año más de 90 millones de toneladas de productos de la combustión del carbón (PCCs), de los que casi el $68 \%$ son

\section{INTRODUCTION}

Over 90 million tonnes of coal combustion products (CCPS) are generated in the European Union (EU-25) yearly, close to $68 \%$ of which in the form of fly ash (1).

(*) Instituto de Ciencias de la Construcción Eduardo Torroja (CSIC), España.

Persona de contacto/Corresponding author: sgoni@ietcc.csic.es (S. Goñi). 
cenizas volantes (1). Como puede verse en la Figura 1, dentro del UE, la utilización de las cenizas volantes en la Industria de la Construcción alcanza actualmente un 47\% del total de su producción. Esta utilización significa: (i) preservar los recursos naturales; (ii) dar un valor añadido a los materiales; (iii) mayor durabilidad al hormigón; y (iiii) añadir un etiquetado verde al mundo de la construcción. Por lo tanto, los gobiernos están favoreciendo, cada vez más, el empleo de materiales secundarios como materia prima para la fabricación de cemento, ya que permite una construcción más sostenible debido a un importante ahorro energético, preservación de las materias primas naturales y reducción de las emisiones de $\mathrm{CO}_{2}$ al medio ambiente.

Actualmente, la industria del cemento emite entre 0,7 a 1,2 toneladas de $\mathrm{CO}_{2}$ por cada tonelada de clínker de cemento, dependiendo del tipo de fuel empleado. En general, el uso de una tonelada de ceniza volante en los cementos de adiciones evita la emisión de una tonelada de $\mathrm{CO}_{2}$. En el año 2003, el empleo de aproximadamente 13,8 millones de toneladas de cenizas en cementos de adición, como materia prima y adición al hormigón, ha supuesto la reducción de alrededor de 11 millones de toneladas $\mathrm{CO}_{2}$ (factor medio de 0,8). Además, cerca de 12 millones de toneladas de materias primas naturales se han salvado, ya que para la producción de 1 tonelada de cemento se emplean 1,6 toneladas de materias primas naturales (1-4).

Los cementos belíticos contribuyen, además, a la reducción de las emisiones de $\mathrm{CO}_{2}$, debido al menor contenido
And as Figure 1 shows, around 47\% of such fly ash is currently reabsorbed by the construction industry. Such reuse helps conserve primary mineral resources, provides a source of valuable raw materials, strengthens the built environment by enhancing concrete durability and makes the construction industry more sustainable. Governments are therefore encouraging the use of secondary raw materials in cement manufacture because for its contribution to energy savings, conservation of natural resources and the reduction of $\mathrm{CO}_{2}$ emissions.

Since the cement industry currently emits from 0.7 to 1.2 tonnes of $\mathrm{CO}_{2}$ per tonne of clinker produced depending on the type of fuel used, for each tonne of cement clinker replaced by fly ash in blended cement one tonne less of $\mathrm{CO}_{2}$ is emitted into the atmosphere. Consequently, the approximately 13.8 million tonnes of fly ash used as a raw material; in blended cement or a concrete admixture in 2003 reduced $\mathrm{CO}_{2}$ emissions by 11 million tonnes (assuming a mean of 0.8 tonnes emitted/tonne of cement). Around 12 million tonnes of raw materials were also saved, inasmuch as it takes 1.6 tonnes of prime materials to manufacture one tonne of cement (1-4).

Belite cements further contributes to lowering $\mathrm{CO}_{2}$ emissions because it has a smaller $\mathrm{CaO}$ content than Portland

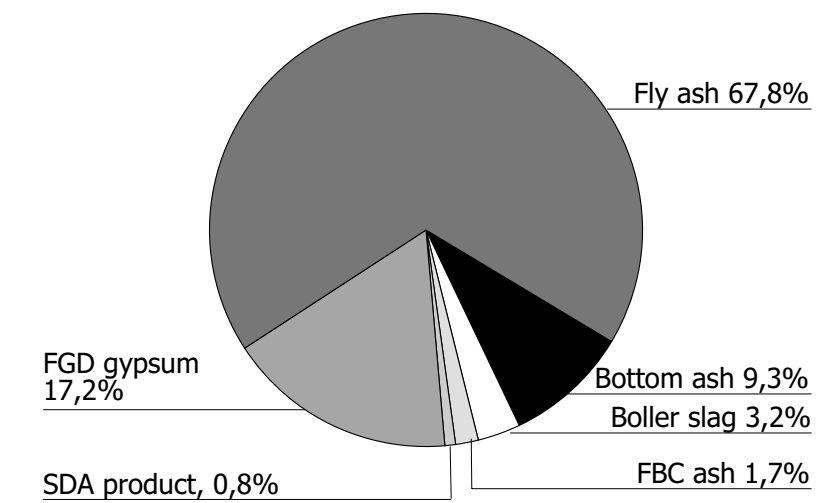

Production of CCPs in Europe (EU 15) in 2003

Total production: 65 million tonnes

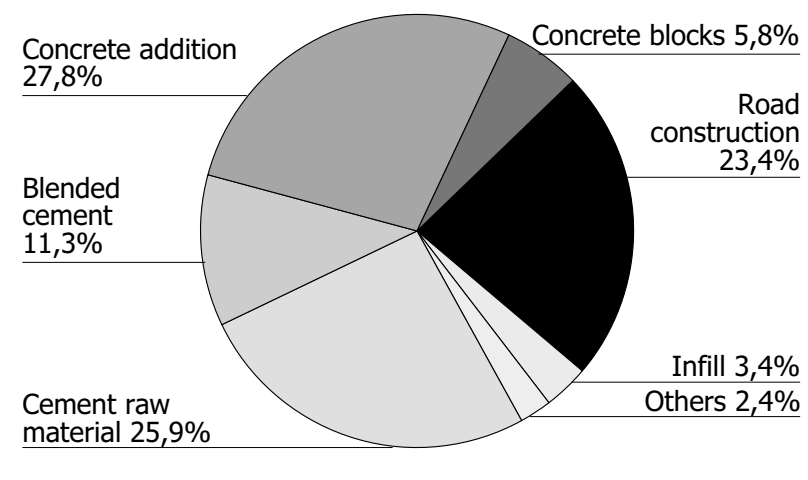

Utilisation of fly ash in the construction industry and underground mining in Europe (EU 15) in 2003 Total utilization: 21.1 million tonnes

Figura 1. Producción de productos de la combustión del carbón (PCCs) y utilización de cenizas volantes en la industria de la construcción europea (EU 15) en el año 2003, de acuerdo con el informe de ECOBA, año 2003 (1).

Figure 1. Coal combustion products (CCPS) and use of fly ash by the European (EU-15) construction industry in 2003, according to ECOBA (2003) (1). 
de $\mathrm{CaO}$ requerido para la formación de la belita $\left(2 \mathrm{CaO} . \mathrm{SiO}_{2}\right)$ en comparación con el cemento Portland tradicional. En este sentido se ha desarrollado por diferentes grupos de investigación una importante familia de cementos belíticos (5-22).

En particular, los cementos belíticos de cenizas volantes (CBCV), obtenidos a partir de cenizas volantes tipo $\mathrm{F}$ y $\mathrm{C}$, están siendo fabricados y caracterizados en nuestro laboratorio siguiendo una ruta hidrotermal-calcinación (23-35). El proceso de fabricación incluye un pretratamiento hidrotermal, donde se obtienen los geles puzolánicos hidratados precursores del cemento. En la segunda etapa, los geles se deshidratan mediante un calentamiento controlado dando lugar al CBCV.

Cuando se utilizan cenizas tipo $\mathrm{F}$ como materia prima, es necesario adicionar una cantidad extra de $\mathrm{CaO}$ comercial para conseguir la relación molar $\mathrm{CaO} / \mathrm{SiO}_{2}$ de 2 (23-28), sin embargo, esta adición puede ser reducida o incluso totalmente evitada si se emplea como materia prima una ceniza volante de alto contenido en cal (tipo C). Este último caso fue estudiado en (30-34), donde una ceniza volante clase $\mathrm{C}$ (de relación molar $\mathrm{CaO} / \mathrm{SiO}_{2}=2$ ) (denominada $\mathrm{CV}$-1) se empleó como única materia prima para sintetizar un cemento belítico denominado CBCV-1.

La optimización de los parámetros de síntesis, incluyendo diferentes temperaturas del proceso hidrotermal $\left(100{ }^{\circ} \mathrm{C}, 150{ }^{\circ} \mathrm{C}\right.$ y $\left.200{ }^{\circ} \mathrm{C}\right)$; agua desmineralizada y disolución de $\mathrm{NaOH} 1 \mathrm{M}$; y la influencia de la temperatura de deshidratación de los precursores en un rango desde $700{ }^{\circ} \mathrm{C}$ a $1.000{ }^{\circ} \mathrm{C}$ fueron publicadas en (30). Los resultados mostraron que el clínker óptimo de cemento belítico se conseguía con una temperatura del tratamiento hidrotermal de la ceniza de $200{ }^{\circ} \mathrm{C}$, donde las fases precursoras obtenidas, tanto en agua como en medio $\mathrm{NaOH}$, fueron: [katoita $\left(\mathrm{C}_{3} \mathrm{ASH}_{4}\right) \quad\left(\mathrm{Ca}_{3} \mathrm{Al}_{2}\right.$ $\left.\left(\mathrm{SiO}_{4}\right)(\mathrm{OH})_{8}\right]$, gel C-S-H $\left(\mathrm{Ca}_{1.5} \mathrm{SiO}_{3.5} \cdot \mathrm{xH}_{2} \mathrm{O}\right)$ y $\alpha-\mathrm{C}_{2} \mathrm{SH}$, junto con calcita $\left(\mathrm{CaCO}_{3}\right)$ y anhidrita $\left(\mathrm{CaSO}_{4}\right)$. Además, cuando el tratamiento hidrotermal se lleva a cabo con disolución de $\mathrm{NaOH}$, se obtiene: aluminio-tobermorita $\left[\mathrm{Ca}_{5} \mathrm{Si}_{5} \mathrm{Al}(\mathrm{OH}) \mathrm{O}_{17} \cdot 5 \mathrm{H}_{2} \mathrm{O}\right]$ y zeolita tipo sodalita $\left[\mathrm{Na}_{6}\left(\mathrm{AlSiO}_{4}\right)_{6} \cdot 4 \mathrm{H}_{2} \mathrm{O}\right]$.

La temperatura óptima de deshidratación de los precursores fue de $800{ }^{\circ} \mathrm{C}$. A dicha temperatura y en el caso del tratamiento hidrotermal con agua, la principal fase cristalina es la belita en su variedad $\alpha{ }^{\prime}{ }_{\mathrm{L}}-\mathrm{Ca}_{2} \mathrm{SiO}_{4}$ junto con restos de calcita, cal libre (CaO) y anhidrita. Dichos resultados indican que no se ha alcanzado el $100 \%$ de reacción, probablemente debido a que el $18 \%$ del $\mathrm{CaO}$ de la ceniza de partida estaba carbonatado (debido a la meteorización durante su almacenamiento en el exterior de la central térmica). cement. For all these reasons, a large family of belite cements is presently under development (5-22).

In particular, fly ash belite cements ( $F A B C$ ) made from class $F$ and $C$ coal fly ash are being synthesized by us via calcination after hydrothermal treatment (23-35). The manufacturing process includes pre-hydrothermal treatment to obtain hydrated pozzolanic cement precursors, which are subsequently dehydrated under controlled heating conditions.

When class $F$ fly ash is used as secondary raw material, additional $\mathrm{CaO}$ is needed to reach a $\mathrm{CaO} / \mathrm{SiO}_{2}$ molar ratio of 2 (23-28); this additional dose may be reduced or even eliminated altogether, however, if class $C$ fly ash is used as the raw material. Studies (30-34) have been conducted on the use of class $\mathrm{C}$ fly ash ( $\mathrm{CaO} / \mathrm{SiO}_{2}$ molar ratio of 2.1: $F A-1$ ) as the secondary raw material for synthesizing an $F A B C$ called $F A B C-1$.

The results on optimal synthesis parameters, including the hydrothermal treatment temperature $\left(100^{\circ} \mathrm{C}, 150^{\circ} \mathrm{C}\right.$ or $200^{\circ} \mathrm{C}$ ), choice of demineralized water or $1 \mathrm{M} \mathrm{NaOH}$ solution and the temperature used for precursor dehydration (ranging from $700{ }^{\circ} \mathrm{C}$ to $1,000{ }^{\circ} \mathrm{C}$ ) were reported in an earlier paper (30). They showed that the optimum belite cement clinker was obtained with a hydrothermal treatment temperature of $200{ }^{\circ} \mathrm{C}$, at which the hydrated cement precursors formed in both water and the $\mathrm{NaOH}$ solution were: [katoite $\left(\mathrm{C}_{3} \mathrm{ASH}_{4}\right)\left(\mathrm{Ca}_{3} \mathrm{Al} \mathrm{C}_{2}\left(\mathrm{SiO}_{4}\right)(\mathrm{OH})_{8}\right.$ ], $\mathrm{C}$-S-H gel $\left(\mathrm{Ca}_{1.5} \mathrm{SiO}_{3.5} \cdot \mathrm{xH}_{2} \mathrm{O}\right)$ and $\alpha-\mathrm{C}_{2} \mathrm{SH}$, as well as calcite $\left(\mathrm{CaCO}_{3}\right)$ and anhydrite $\left.\left(\mathrm{CaSO}_{4}\right)\right]$. When the solution used in hydrothermal treatment was $\mathrm{NaOH}$, aluminum-tobermorite $\left[\mathrm{Ca}_{5} \mathrm{Si}_{5} \mathrm{Al}(\mathrm{OH}) \mathrm{O}_{17} .5 \mathrm{H}_{2} \mathrm{O}\right]$ and zeolite $\left(\mathrm{Na}_{6}\left[\mathrm{AlSiO}_{4}\right]_{6} \cdot 4 \mathrm{H}_{2} \mathrm{O}\right)$-type sodalite were also formed.

The optimal heating temperature for hydrated precursors was found to be $800^{\circ} \mathrm{C}$. The main crystalline phase formed in water at this temperature was belite of the $\alpha^{\prime}{ }^{\prime}-\mathrm{Ca}_{2} \mathrm{SiO}_{4}$ variety, along with some unreacted free lime ( $\mathrm{CaO})$ and anhydrite. These results indicate that the degree of reaction was under $100 \%$, most likely because $18 \%$ of the initial $\mathrm{CaO}$ in the fly ash was carbonated (due to the weathering taking place during outdoor storage at the power plant). 
Cuando el tratamiento hidrotermal se llevó a cabo con disolución de $\mathrm{NaOH} 1 \mathrm{M}$ y posterior calentamiento a $800{ }^{\circ} \mathrm{C}$, se observó un aumento importante de la reactividad de la ceniza de partida, duplicando prácticamente la cantidad de $\alpha{ }^{\prime}{ }_{\mathrm{L}}-\mathrm{Ca}_{2} \mathrm{SiO}_{4}$ respecto al formado cuando en el tratamiento hidrotermal se empleó agua desmineralizada (ver Figuras 7 y 10 de la referencia 30).

A temperaturas superiores a $800{ }^{\circ} \mathrm{C}$, se forma gehlenita y cal libre (CaO). Ambas fases no son deseables ya que la gehlenita tiene poca reactividad hidráulica y la cal libre produce expansión durante la hidratación. Es más, la fase $\alpha^{\prime}{ }_{\mathrm{L}}-\mathrm{Ca}_{2} \mathrm{SiO}_{4}$ se convierte parcialmente en $\beta-\mathrm{Ca}_{2} \mathrm{SiO}_{4}$ y se forman trazas de mayenita $\left(\mathrm{Ca}_{12} \mathrm{~A}_{14} \mathrm{O}_{33}\right)$.

La microestructura y propiedades mecánicas del cemento optimizado CBCV-1 obtenido después del tratamiento hidrotermal en agua (CBCV-1-A) y en disolución de $\mathrm{NaOH}(\mathrm{CBCV}-1-\mathrm{S})$ junto con la evolución de la microestructura de la ceniza de partida en cada uno de los pasos del proceso de síntesis se ha estudiado y los resultados presentados en (33 y 34). La resistencia a compresión de probetas de mortero fabricadas con el cemento CBCV-1-A y CBCV-1-S fueron muy bajas a primeras edades y curado en condiciones normales (temperatura ambiente y humedad relativa $>95 \%$ ). Sin embargo, la presencia de un $2 \%$ de un aditivo superplastificante reductor de la demanda de agua y un curado a $40{ }^{\circ} \mathrm{C}$ con una humedad relativa $>95 \%$ supone un aumento de la resistencia a compresión de un orden de magnitud, alcanzado valores de $11.4 \mathrm{MPa}$ después de 7 días y $15 \mathrm{MPa}$ después de 28 días de curado del mortero fabricado con cemento CBCV-1-A. En el caso del curado acelerado a $40{ }^{\circ} \mathrm{C}$, el cemento CBCV-1-A tiene valores de resistencia mecánica similares a los que presentan los cementos naturales -CNR 4, CNR 8, CNL 8y los de albañilería - MC 5 y MC $12.5 X-$, según la norma española de cementos. El cemento tipo CBCV-1-S presenta valores similares a los requeridos para los cementos CNR 4 y MC 5.

La composición de la ceniza volante tipo C presenta una gran variabilidad, por ello actualmente estamos estudiando cómo este hecho puede afectar al cemento belítico final (CBCV). En este trabajo se presentan los estudios de un cemento belítico, denominado CBCV-2-A, que ha sido obtenido a partir de otro tipo de ceniza volante tipo C Ilamada CV-2. La influencia de las condiciones de curado y el aditivo reductor de la demanda de agua en la actividad hidráulica del material se ha evaluado durante un periodo de 180 días desde el amasado, mediante la evolución del contenido de agua combinada y el grado de avance de la hidratación. La resistencia mecánica, porosidad y distribución de tamaño de poro se ha estudiado en probetas semejantes pero de mortero.
Where the fly ash was treated in a $1 \mathrm{M} \mathrm{NaOH}$ solution and subsequently heated at $800^{\circ} \mathrm{C}$, the reactivity of the initial FA-1 was much higher, nearly doubling the amount of $\alpha^{\prime}{ }_{L}-\mathrm{Ca}_{2} \mathrm{SiO}_{4}$ formed when the treatment medium was demineralized water (see Figures 7 and 10, reference 30).

Gehlenite and free lime ( $\mathrm{CaO}$ ) formed at temperatures over $800^{\circ} \mathrm{C}$. Neither is desirable, for hydraulic activity is low in the former and the latter may prompt expansion during hydration. Moreover, some of the $\alpha^{\prime}{ }_{L}-\mathrm{Ca}_{2} \mathrm{SiO}_{4}$ converted to $\beta-\mathrm{Ca}_{2} \mathrm{SiO}_{4}$ and traces of mayenite $\left(\mathrm{Ca}_{12} \mathrm{~A}_{14} \mathrm{O}_{33}\right)$ formed.

The microstructure and mechanical properties of the optimal FABC-1 obtained after hydrothermal treatment in water (FABC-1-W) and $\mathrm{NaOH}$ solution (FABC-1-N), along with the microstructure of the initial $F A-1$, have been studied (33) at every step of $F A B C-1$ synthesis $(33,34)$. Early age compressive strength of both FABC-1-W and $F A B C-1-N$ mortars was very low when specimens were cured under standard conditions. When a superplasticizing additive was included at a rate of $2 \%$ and the mortars were cured at $40{ }^{\circ} \mathrm{C}$ and $>95 \% \mathrm{RH}$, however, compressive strength increased by one order of magnitude, with FABC-1-W mortars reaching 11.4 MPa after seven days and $15 \mathrm{MPa}$ after 28. When subjected to $40{ }^{\circ} \mathrm{C}$ accelerated curing, the FABC-1-W cement met the mechanical requirements established for the following Spanish special cements: natural cements CNR 4, CNR 8, CNL 8 and masonry cements MC 5 and MC 12.5X. FABC$1-N$ met the requirements for CNR 4 and MC 5.

In light of the compositional variability of class $C$ coal fly ash, the effect of its composition on fly ash belite cement $(F A B C)$ is currently under study. Research on FABC-2-W, obtained from another type of class $C$ fly ash (FA-2) is discussed in the present report. The effect of curing and a water-reducing admixture on the hydraulic activity of this cement was evaluated over a period of 180 days after mixing by determining the bound water content and the degree of hydration. Mechanical strength, porosity and pore-size distribution were also studied in equivalent mortar samples. 


\section{PROCEDIMIENTO EXPERIMENTAL}

\subsection{Materiales}

Como materia prima se ha empleado una ceniza volante española, denominada CV-2, de alto contenido en cal, tipo $\mathrm{C}$, según la norma EN-UNE $450\left(\mathrm{SiO}_{2}+\mathrm{Al}_{2} \mathrm{O}_{3}+\mathrm{Fe}_{2} \mathrm{O}_{3}\right.$ $<70 \%$ ); con una relación molar $\mathrm{CaO} / \mathrm{SiO}_{2}=1$. En la Tabla 1 se da la composición química de la ceniza, obtenida de acuerdo con la norma española UNE-EN 196-2.

La composición mineralógica de la ceniza aparece en la Figura 2. Los principales compuestos cristalinos son: cal libre $(\mathrm{CaO})$, anhidrita $\left(\mathrm{CaSO}_{4}\right)$, cuarzo $\left(\mathrm{SiO}_{2}\right)$, hematita $\left(\mathrm{Fe}_{2} \mathrm{O}_{3}\right)$, magnetita $\left(\mathrm{Fe}_{3} \mathrm{O}_{4}\right)$ y mullita $\left(3 \mathrm{Al}_{2} \mathrm{O}_{3} \cdot 2 \mathrm{SiO}_{2}\right)$; el halo amorfo que se aprecia entre 15 y 35 grados de la zona angular $2 \theta$ corresponde a la parte vítrea de la ceniza, principalmente $\mathrm{SiO}_{2}$.

\subsection{Procedimiento}

El proceso de fabricación del cemento belítico de cenizas volantes, denominado CBCV-2-A, tiene lugar en dos etapas. En la primera, una mezcla de CV-2 y $\mathrm{CaO}$ comercial, con una relación molar $\mathrm{Ca} / \mathrm{Si}$ de 2, se trata hidrotermalmente con agua desmineralizada en una proporción agua:sólido de 3:1; a una temperatura de

\section{EXPERIMENTAL PROCEDURE}

\subsection{Materials}

A high Ca content coal fly ash (ASTM class C) denominated FA-2 was used as a raw material. The chemical composition of $F A-2$, determined as specified in Spanish standard UNE-EN 196-2, is given in Table 1. FA-2 meets the requirements for ASTM class $C$ cement and EN-UNE 450 specifications: its $\mathrm{SiO}_{2}+\mathrm{Al}_{2} \mathrm{O}_{3}+\mathrm{Fe}_{2} \mathrm{O}_{3}$ content is lower than $70 \%$ and it has a high $\mathrm{CaO}$ content, with a $\mathrm{CaO} / \mathrm{SiO}_{2}$ molar ratio of 1 .

The mineralogical composition of FA-2 is given in Figure 2. The crystalline compounds are free lime ( $\mathrm{CaO})$, anhydrite $\left(\mathrm{CaSO}_{4}\right)$, quartz $\left(\mathrm{SiO}_{2}\right)$, hematite $\left(\mathrm{Fe}_{2} \mathrm{O}_{3}\right)$, magnetite $\left(\mathrm{Fe}_{3} \mathrm{O}_{4}\right)$ and mullite $\left(\mathrm{Al}_{6} \mathrm{Si}_{2} \mathrm{O}_{13}\right)$. The amorphous halo between $2 \theta$ values of 15 and 35 degrees corresponds to the glassy component in the fly ash, mainly $\mathrm{SiO}_{2}$.

\subsection{Procedure}

The manufacture of fly ash belite cement, here FABC-2$W$, was a two-step process. First, FA-2 and commercial $\mathrm{CaO}(\mathrm{Ca} / \mathrm{Si}$ molar ratio $=2)$ were mixed with demineralized water (solution:solid ratio of 3:1) and continuously stirred for four hours at a temperature of $200{ }^{\circ} \mathrm{C}$ and a pressure of $1.24 \mathrm{MPa})$. After drying ( $80^{\circ} \mathrm{C}$ overnight),

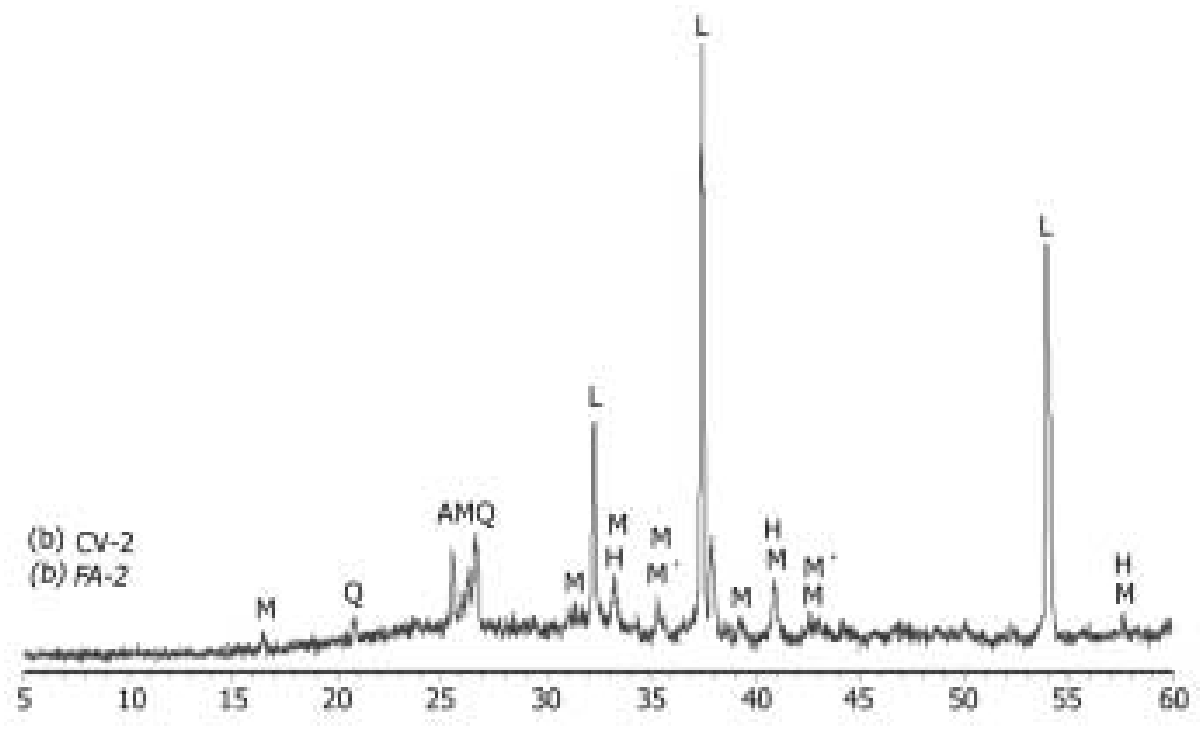

[20]

A anhidrita; Qcuarzo; M mullita; $M^{\prime}$ magnetita; $L$ arcilla; $H$ hematita

A arhydhte; $Q$ quartz; $M$ mulve; $M^{*}$ magnetite; $L$ lime; H hematite

Figura 2. Difractograma de rayos $X$ de la ceniza volante de partida (CV-2).

Figure 2. $X$-ray diffractogram of the starting fly ash (FA-2). 
$200{ }^{\circ} \mathrm{C}$ y $1,24 \mathrm{MPa}$ de presión durante 4 horas y agitación continua. En la segunda etapa, el sólido seco (80 ${ }^{\circ} \mathrm{C}$ durante una noche) se calienta a $600^{\circ} \mathrm{C}$ con una velocidad de $10^{\circ} \mathrm{C} / \mathrm{min}$, y después de $600^{\circ} \mathrm{C}$ a $800{ }^{\circ} \mathrm{C}$ con una velocidad de $5^{\circ} \mathrm{C} / \mathrm{min}$.

Se prepararon probetas de pasta de $1 \times 1 \times 6 \mathrm{~cm}$ con agua desmineralizada, en una proporción agua/cemento de 0,68 y un aditivo comercial reductor de la demanda de agua (Rheobuild-1000) en una proporción de 2\% (por peso de cemento). Las pastas se curaron a $21^{\circ} \mathrm{C}$ (curado normal) y a $40{ }^{\circ} \mathrm{C}$ con una humedad relativa $>95 \%$ durante un periodo de 180 días desde el amasado. En el caso de las resistencias mecánicas y los análisis de porosidad, se emplearon probetas normalizadas de mortero,

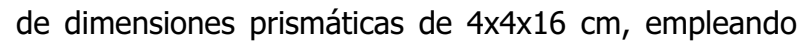
un $100 \%$ de CBCV-2, con una relación arena/cemento 3:1 y agua/cemento de 1, según la norma española UNEEN 196-1 (con algunas modificaciones). Después de dos días, una serie de probetas de mortero se desmoldaron y curaron a $21^{\circ} \mathrm{C}$ (curado normal) y una humedad relativa $>95 \%$; una segunda serie de probetas de mortero fueron preparadas con un aditivo comercial reductor de agua (Rheobuild-1000) en una proporción de un $2 \%$ (en peso de cemento, cantidad requerida para este tipo de material) y curado a $21^{\circ} \mathrm{C}$ y $40^{\circ} \mathrm{C}$ en atmósfera saturada de humedad, en este caso la relación agua/cemento fue de 0,85 .

\subsection{Equipamiento}

El tratamiento hidrotermal ha sido llevado a cabo con un equipo Parr modelo 4522 de 1 litro de capacidad dotado de un controlador de temperatura PID modelo 4842. El análisis térmico se ha realizado empleando un equipo Netzsch con un sistema de análisis simultáneo STA 409 utilizando $50 \mathrm{mg}$ de muestra en cada análisis y corriente dinámica de nitrógeno (velocidad de flujo $=100 \mathrm{~cm}^{3} / \mathrm{min}$ ), con una velocidad de calentamiento de $10{ }^{\circ} \mathrm{C} / \mathrm{min}$. Los análisis de difracción de rayos $X$ se han realizado con un equipo Philips PW 1730 con cámara de grafito y una radiación de $\mathrm{Cu} \mathrm{K} \alpha_{1}$. Para el estudio de la porosidad y distribución de tamaño de poro se ha empleado un porosímetro de intrusión de mercurio de la casa Micromeritics Pore Sizer IV $9600 \mathrm{~V} 1.05$, en el rango de presiones comprendido entre 0,5 y 33.000 psia.

\section{RESULTADOS Y DISCUSIÓN}

\subsection{Difracción de rayos $X$ y análisis térmico}

El cemento belítico CBCV-2-A se ha obtenido por calentamiento a $800{ }^{\circ} \mathrm{C}$ de las fases precursoras de dicho material. En la Tabla 1 se recoge la composición química de dicho cemento. the solid was heated to $600^{\circ} \mathrm{C}$ at a rate of $10^{\circ} \mathrm{C} / \mathrm{min}$ and then to $800^{\circ} \mathrm{C}$ at $5^{\circ} \mathrm{C} / \mathrm{min}$.

Pastes were prepared with a water/cement ratio of 0.68 and contained $2 \%$ (by cement weight) of a commercial water-reducing additive (Rheobuild-1000). The pastes were cured at $21^{\circ} \mathrm{C}$ (standard curing) or $40^{\circ} \mathrm{C}$ at $>95 \%$ $\mathrm{RH}$ for 180 days. For mechanical strength and porosity tests, $4 \times 4 \times 16-\mathrm{cm}$ mortar specimens were prepared as specified in Spanish standard UNE-EN 196-1 (with some modifications), with $100 \%$ FABC-2, a water/cement ratio of 1 and a sand/cement ratio of 3:1.The specimens were demoulded after two days and cured at $>95 \% \mathrm{RH}$ and $21^{\circ} \mathrm{C}$. A second series was prepared with $2 \%$ (by cement weight, as required for this type of cement) of a commercial water-reducing additive (Rheobuild-1000) and a water/cement ratio of 0.85 . These specimens were cured at $21^{\circ} \mathrm{C}$ or $40^{\circ} \mathrm{C}$ in a water-saturated atmosphere.

\subsection{Equipment}

Hydrothermal treatments were performed with a Parr 4522 unit (1000-ml pump with a split ring closure and a PID 4842 thermostat). Thermal analyses were recorded on a Netzsch facility fitted with an STA 409 simultaneous analysis system providing a nitrogen stream at $100 \mathrm{~cm}^{3} / \mathrm{min}$. The $50-\mathrm{mg}$ samples were heated at a rate of $10^{\circ} \mathrm{C} / \mathrm{min}$. XRD patterns were recorded on a Philips $P W$ 1730 diffractometer equipped with a graphite monochro-

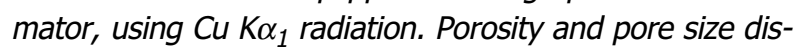
tribution were determined with mercury intrusion porosimetry, conducted with a Micrometrics IV 9600 V1.05 pore sizer over a pressure range of 0.5-33,000 psia.

\section{RESULTS AND DISCUSSION}

\subsection{X-ray diffraction and thermal analyses}

FABC-2-W was obtained by heating the precursor phases to $800{ }^{\circ} \mathrm{C}$. The chemical composition of this cement is given in Table 1. 
Tabla 1 / Table 1

Composición química de la ceniza de partida (CV-2) y del cemento belítico CBCV-2-A (\% en peso) Chemical composition of the starting fly ash, FA-2, and FABC-2-W (\% by weight)

\begin{tabular}{|c|c|c|c|c|c|c|c|c|c|c|c|}
\hline & $\begin{array}{l}\text { PF* } \\
\text { LOI* } \\
\end{array}$ & $\begin{array}{l}\mathrm{CaO} \\
\mathrm{CaO} \\
\end{array}$ & $\begin{array}{l}\mathrm{SiO}_{2} \text { (total) } \\
\mathrm{SiO}_{2} \text { (total) } \\
\end{array}$ & $\begin{array}{l}\mathrm{Fe}_{2} \mathrm{O}_{3} \\
\mathrm{Fe}_{2} \mathrm{O}_{3} \\
\end{array}$ & $\begin{array}{l}\mathrm{Al}_{2} \mathrm{O}_{3} \\
\mathrm{Al}_{2} \mathrm{O}_{3} \\
\end{array}$ & $\begin{array}{l}\text { MgO } \\
M g O \\
\end{array}$ & $\begin{array}{l}\mathrm{SO}_{3} \\
\mathrm{SO}_{3} \\
\end{array}$ & $\begin{array}{l}\mathrm{Na}_{2} \mathrm{O} \\
\mathrm{Na}_{2} \mathrm{O} \\
\end{array}$ & $\begin{array}{l}\mathbf{K 2 O} \\
K 2 O \\
\end{array}$ & $\begin{array}{l}\mathrm{SiO}_{2}{ }^{\&} \text { (reactiva) } \\
\mathrm{SiO}_{2}^{\&} \text { (reactive) }\end{array}$ & $\begin{array}{l}\text { BET }\left(\mathrm{m}^{2} / \mathrm{gr}\right) \\
\text { BET }\left(\mathrm{m}^{2} / g r\right) \\
\end{array}$ \\
\hline $\begin{array}{l}\text { CV-2 } \\
F A-2 \\
\end{array}$ & 4.0 & 32.0 & 32.8 & 4.2 & 19.3 & 2.2 & 2.8 & 0.44 & 1.6 & 25.2 & 3 \\
\hline $\begin{array}{l}\text { CBCV-2 A } \\
F A B C-2 W\end{array}$ & 1.4 & 48.3 & 28.7 & 2.3 & 15.2 & 1.4 & 1.7 & 0.25 & 0.5 & 28.6 & 6.4 \\
\hline
\end{tabular}

* PF = pérdida al fuego; \&Sílice reactiva de acuerdo a la norma española UNE-80-224.

* LOI = loss on ignition; \&Silica reactivity as per Spanish standard UNE-80-224.

En la Figura 3 se presentan los difractogramas del cemento de partida, así como el de las pastas hidratadas a $21^{\circ} \mathrm{C}$ (curado normal) y a $40^{\circ} \mathrm{C}$. En el caso del cemento anhidro CBCV-2-A [Figura 3 (a)], se aprecian unas reflexiones anchas entre 32-33 de la zona angular $2 \theta$, que corresponden a la variedad cristalográfica $\alpha^{\prime}{ }_{\mathrm{L}}-\mathrm{C}_{2} \mathrm{~S}$ de la belita $\left(\mathrm{Ca}_{2} \mathrm{SiO}_{4}\right)$ pobremente cristalizada; gelenita $\left(\mathrm{Ca}_{2} \mathrm{Al}_{2} \mathrm{SiO}_{7}\right)$ y trazas de mayenita $\left(\mathrm{Ca}_{12} \mathrm{Al}_{14} \mathrm{O}_{33}\right)$. La ausencia de cal libre sugiere el $100 \%$ de reacción. El contenido de calcita (determinado a partir del análisis termogravimétrico) es de $1,1 \%$.

En el caso del curado normal, los principales compuestos hidratados son: $\mathrm{C}_{2} \mathrm{SH}_{0,35}\left(\mathrm{Ca}_{2} \mathrm{SiO}_{4} \cdot 0,35 \mathrm{H}_{2} \mathrm{O}\right)$ [Figura 3 (b)], que aumenta progresivamente con el tiempo; monosulfoaluminato de calcio hidratado $\left[\mathrm{Ca}_{4} \mathrm{Al}_{2}\left(\mathrm{SO}_{4}\right) \mathrm{O}_{6} \cdot 10 \mathrm{H}_{2} \mathrm{O}\right]$ y stratlingita $\left(\mathrm{Ca}_{2} \mathrm{Al}_{2} \mathrm{SiO}_{7} \cdot 8 \mathrm{H}_{2} \mathrm{O}\right)$ (formada a partir de la hidratación de la gelenita), estas dos últimas de la familia estructural tipo AFm (36), y que aparecen después de 90 y 180 días de hidratación, respectivamente [Figura 3 (c y d)]. También se forman gel C-S-H $\left(\mathrm{Ca}_{1,5} \mathrm{SiO}_{3,5} \cdot \mathrm{xH}_{2} \mathrm{O}\right)$ [aparecen las tres únicas reflexiones de este compuesto, según estudios de Taylor (36)] y trazas de portlandita $\mathrm{Ca}(\mathrm{OH})_{2}$.

Las principales diferencias producidas cuando las pastas se curan a $40{ }^{\circ} \mathrm{C}$ son: la formación de katoita $\left[\mathrm{Ca}_{3} \mathrm{Al}_{2}\left(\mathrm{SiO}_{4}\right)(\mathrm{OH})_{8}\right]$ después de 90 días y desaparece la portlandita [ver Figura 3 (c y d)]; la ausencia de stratlingita; la formación del monocarbo-aluminato de calcio hidratado $\left[\mathrm{Ca}_{4} \mathrm{Al}_{2} \mathrm{O}_{6}\left(\mathrm{CO}_{3}\right) \cdot 11 \mathrm{H}_{2} \mathrm{O}\right]$, probablemente a partir de la carbonatación del $\mathrm{Ca}_{4} \mathrm{Al}_{2}\left(\mathrm{SO}_{4}\right) \mathrm{O}_{6} \cdot 10 \mathrm{H}_{2} \mathrm{O}$, y la disminución de la fase $\mathrm{C}_{2} \mathrm{SH}_{0,35}$, detectada claramente por la disminución de la intensidad de la reflexión centrada a 31 grados de la zona angular $2 \theta$.

La evolución con el tiempo del grado de hidratación, semicuantitativo $(\alpha)$ del $\alpha{ }^{\circ}{ }_{\mathrm{L}}-\mathrm{C}_{2} \mathrm{~S}$ (calculado a partir de la intensidad de la reflexión a 41,1 grados de la zona angular 20) para las dos condiciones de curado aparece en la Figura 4. No ha sido posible emplear la reflexión de máxima intensidad del $\alpha^{{ }}{ }_{\mathrm{L}}-\mathrm{C}_{2} \mathrm{~S}$ que aparece en 32,5
The XRD patterns for the anhydrous $F A B C-2-W$ and the hydrated pastes cured at $21^{\circ} \mathrm{C}$ (standard) and $40^{\circ} \mathrm{C}$ are shown in Figure 3.The diffractograms for the former [Figure 3(a)] have wide peaks at $2 \theta$ values of 32-33 degrees, attributed to $\alpha^{\prime}{ }_{L}-C_{2} S$ the poorly crystallized variety of belite; gehlenite $\left(\mathrm{Ca}_{2} \mathrm{Al}_{2} \mathrm{SiO}_{7}\right)$ and traces of mayenite $\left(\mathrm{Ca}_{12} \mathrm{Al}_{14} \mathrm{O}_{33}\right)$ are also visible; the absence of free lime suggests that the initial materials reacted fully. Thermogravimetric analysis showed the calcite content to be $1.1 \%$.

The hydrated compounds formed under standard curing included $\mathrm{C}_{2} \mathrm{SH}_{0.35}\left(\mathrm{Ca}_{2} \mathrm{SiO}_{4} \cdot 0.35 \mathrm{H}_{2} \mathrm{O}\right)$ [Figure 3(b)] in amounts that increased with hydration time, hydrated calcium-monosulpho-aluminate $\left[\mathrm{Ca}_{4} \mathrm{Al}_{2}\left(\mathrm{SO}_{4}\right) \mathrm{O}_{6} \cdot 10 \mathrm{H}_{2} \mathrm{O}\right]$ and stratlingite $\left(\mathrm{Ca}_{2} \mathrm{Al}_{2} \mathrm{SiO}_{7} 8 \mathrm{H}_{2} \mathrm{O}\right)$, a product of gehlenite hydration. The latter two, of the AFm structural family (36), appeared after 90 and 180 days, respectively [Figure 3 ( $\mathrm{C}$ and d)]. $\mathrm{C}-\mathrm{S}-\mathrm{H}$ gel $\left(\mathrm{Ca}_{1.5} \mathrm{SiO}_{3.5} \mathrm{XH}_{2} \mathrm{O}\right)$ and traces of portlandite $\left[\mathrm{Ca}(\mathrm{OH})_{2}\right]$ also formed.

The chief differences observed when the hydrated pastes were cured at $40^{\circ} \mathrm{C}$ were the formation of katoite $\left[\mathrm{Ca}_{3} \mathrm{Al}_{2}\left(\mathrm{SiO}_{4}\right)(\mathrm{OH})_{8}\right]$, which appeared after 90 days and increased thereafter, the disappearance of portlandite [see Figure 3 ( $c$ and d)], the absence of stratlingite, the formation of hydrated calcium-monocarbo-aluminate $\left[\mathrm{Ca}_{4} \mathrm{Al}_{2} \mathrm{O}_{6}\left(\mathrm{CO}_{3}\right) \cdot 11 \mathrm{H}_{2} \mathrm{O}\right]$, perhaps as a result of $\mathrm{Ca}_{4} \mathrm{Al}_{2}\left(\mathrm{SO}_{4}\right) \mathrm{O}_{6} \cdot 10 \mathrm{H}_{2} \mathrm{O}$ carbonation, and the smaller amount of $\mathrm{C}_{2} \mathrm{SH}_{0.35}$, clearly visible from the intensity of the signal centred over a $2 \theta$ value of 31 degrees.

A semi-quantitative measure of the degree of hydration of $\alpha{ }^{~} L-C_{2} S$ (calculated from the intensity of the peak at 41.1 degrees) is shown in Figure 4.The largest peak, recorded at 32.5 degrees and attributed to $\alpha^{\prime}{ }_{L}-C_{2} S$, could not be used due to the existence of overlapping signals corresponding to hydration products such as 


\section{PASTA / PASTE}

normal + aditwo / standard + additive

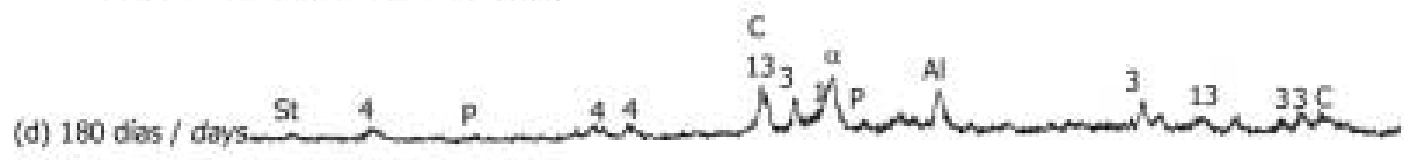

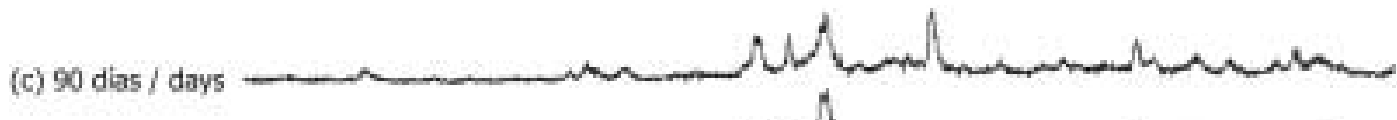

(b) 7 dias / days

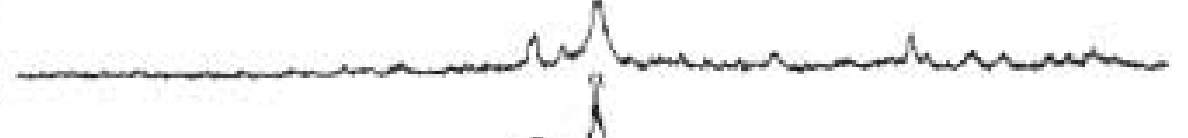

(a) 0 dias / day

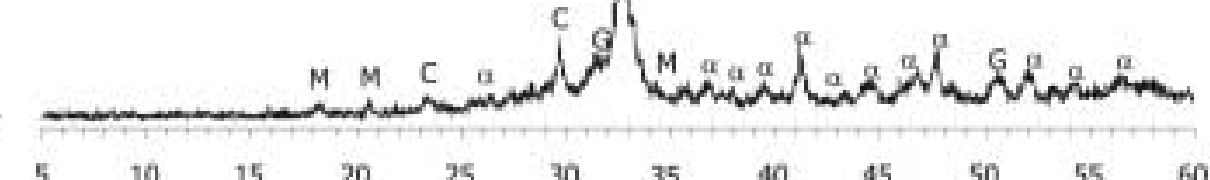

[20]

PASTA / PASTE

$40 \circ \mathrm{C}+$ aditiwo / $40 \varnothing \mathrm{C}+$ additive

C

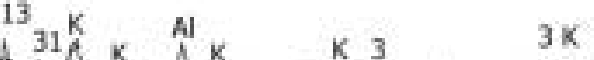

(d)180 dias / days
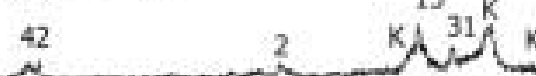

K K K 3

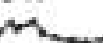

(C) 90 dias / dorys

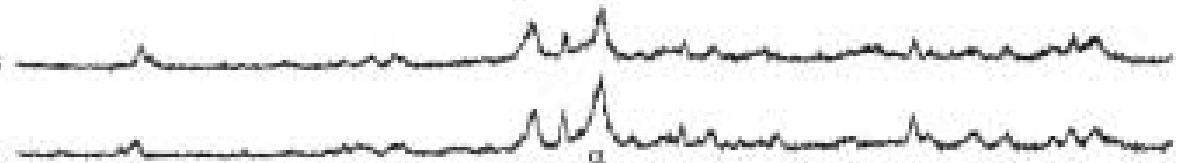

(b) 7 dlas / days
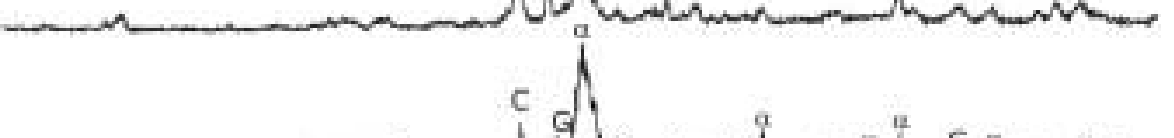

(a) 0 dias / doy
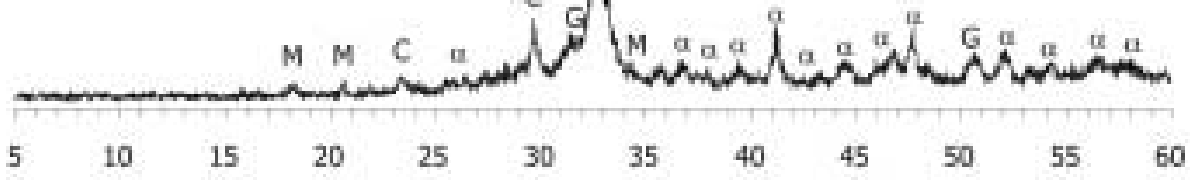

[2甘]

$\mathrm{C}=$ calcita / calcite; $\alpha \alpha^{\prime}{ }_{\mathrm{L}}$-C2C; $\mathrm{M}=$ mayetina / mayetine; $\mathrm{G}=$ gelenita / gehlenite; $\mathrm{K}$ = katoita / katoite; $1 \mathrm{Ca}_{1.5} \mathrm{SiO}_{3.5} \cdot \mathrm{xH}_{2} \mathrm{O}$;

$2 \mathrm{C}_{4} \mathrm{AcH}_{11} ; 3 \mathrm{C}_{2} \mathrm{SH}_{0.35} ; 4$ monosultoaluminato $\mathrm{C}_{4} \mathrm{AsH}_{10} / 4$ monosulfo $\mathrm{C}_{4} A s H_{10} ;$ St = stratlingita / stratlingite;

$\mathrm{P}=$ portlandita / portlandite; $\mathrm{Al}=$ aluminio / aluminium

Figura 3. Difractograma de rayos $X$ de la pasta hidratada CBCV-2-A. Influencia del curado.

Figure 3. X-ray diffractogram of hydrated FABC-2-W paste. Effect of curing conditions.

de la zona angular $2 \theta$, debido al solapamiento con otros productos de hidratación como es la katoita y el gel C-S-H, que también producen reflexiones en esa zona. Como se puede observar, la hidratación es más rápida cuando el curado es a $40{ }^{\circ} \mathrm{C}$, duplicando prácticamente los valores del grado de hidratación cuando el curado se realiza a temperatura ambiente. La hidratación total se alcanza después de 90 días. La hidratación es más lenta en el caso del curado a temperatura ambiente, alcanzando un valor del grado de hidratación de 0,6 a 180 días. El grado de hidratación aumenta con el tiempo según una función logarítmica para ambas condiciones de curado. katoite and C-S-H gel. Hydration took place more rapidly when specimens were cured at $40^{\circ} \mathrm{C}$, with the degree of hydration doubling the findings for standard curing. Full hydration was reached after 90 days, whereas under standard conditions the degree of hydration reached after 180 days was 0.6. Degree of hydration increased logarithmically for both curing conditions. 
El contenido de agua combinada se ha calculado a partir de los análisis termogravimétricos de las pastas, que fueron secadas previamente a temperatura ambiente hasta peso constante en desecadores con gel de sílice, para eliminar el agua evaporable. A modo de ejemplo en la Figura 5 se presentan los perfiles de las curvas de TG y DTG de la pasta de cemento CBCV-2-A hidratada durante 180 días y la evolución del agua combinada con el tiempo. Se produce una pérdida de peso entre $25^{\circ} \mathrm{C}$ y $700^{\circ} \mathrm{C}$ en la curva TG y un pico con dos máximos en $100{ }^{\circ} \mathrm{C}$ y $200{ }^{\circ} \mathrm{C}$ en la correspondiente curva DTG, debidos a la pérdida de las moléculas de agua de los productos hidratados, antes mencionados. La segunda pérdida de peso entre 700-900 ${ }^{\circ} \mathrm{C}$ produce un pico centrado a $800{ }^{\circ} \mathrm{C}$, que es debido a la eliminación de las moléculas de $\mathrm{CO}_{2}$ procedentes de la descomposición de la $\mathrm{CaCO}_{3}$ (parte del contenido de calcita procede del cemento anhidro).

La pérdida de peso debido a la descomposición de la calcita no ha sido tenida en cuanta para calcular el contenido de agua combinada. En el caso del curado a temperatura ambiente, el contenido en agua combinada aumenta con el tiempo siguiendo una función logarítmica, alcanzado un valor del $21 \%$ a 180 días de hidratación (ver Figura 5). Cuando el curado se realiza a $40^{\circ} \mathrm{C}$, la ganancia de agua combinada es mayor a primeras edades, y disminuye después de 28 días de hidratación, alcanzando después de 90 días valores similares al ensayo llevado a cabo a temperatura ambiente. Este fenómeno parece indicar que la temperatura de curado podría provocar alguna conversión de los compuestos hidratados iniciales, quizás de la fase hexagonal stratligita a otras con menos agua en su estructura, tales como la fase cúbica katoita, de acuerdo a la siguiente ecuación:
The combined water content was determined with thermogravimetric techniques. The pastes were first dried at room temperature with silica gel desiccators to eliminate the evaporable water. Examples of TG and DTG curves -for 180-day hydrated FABC-2-W pastes and combined water- are shown in Figure 5. A weight loss can be seen between $25^{\circ} \mathrm{C}$ and $700^{\circ} \mathrm{C}$ on the TG curve and the peak with two maxima -at $100^{\circ} \mathrm{C}$ and $200^{\circ} \mathrm{C}$ - on the respective DTG curve, which reflects the release of water molecules from the hydrated products. A second weight loss between $700{ }^{\circ} \mathrm{C}$ and $900^{\circ} \mathrm{C}$, represented as a peak centred at $800{ }^{\circ} \mathrm{C}$, was due to the release of $\mathrm{CO}_{2}$ molecules resulting from the decomposition of $\mathrm{CaCO}_{3}$ (some of the calcite was present in the initial anhydrous FABC-2-W).

The weight loss due to calcite decomposition was not considered when calculating the combined water content, which under standard curing increased logarithmically to a value of $21 \%$ after 180 days of hydration (Figure 5). When specimens were cured at $40{ }^{\circ} \mathrm{C}$, the combined water content grew faster at earlier ages than in specimens cured under standard conditions, but the rate tapered after 28 days and the values reached after 90 days were similar for both types of curing. This would indicate that the curing temperature may prompt the conversion of the initial hydrated compounds - perhaps the hexagonal stratlingite- into other phases such as katoite with smaller amounts of combined water molecules, according to the following reaction:

$$
\mathrm{Ca}_{2} \mathrm{Al}_{2} \mathrm{SiO}_{7} \cdot 8 \mathrm{H}_{2} \mathrm{O}+\mathrm{Ca}(\mathrm{OH})_{2} \rightarrow \mathrm{Ca}_{3} \mathrm{Al}_{2}\left(\mathrm{SiO}_{4}\right)(\mathrm{OH})_{8}+5 \mathrm{H}_{2} \mathrm{O}
$$

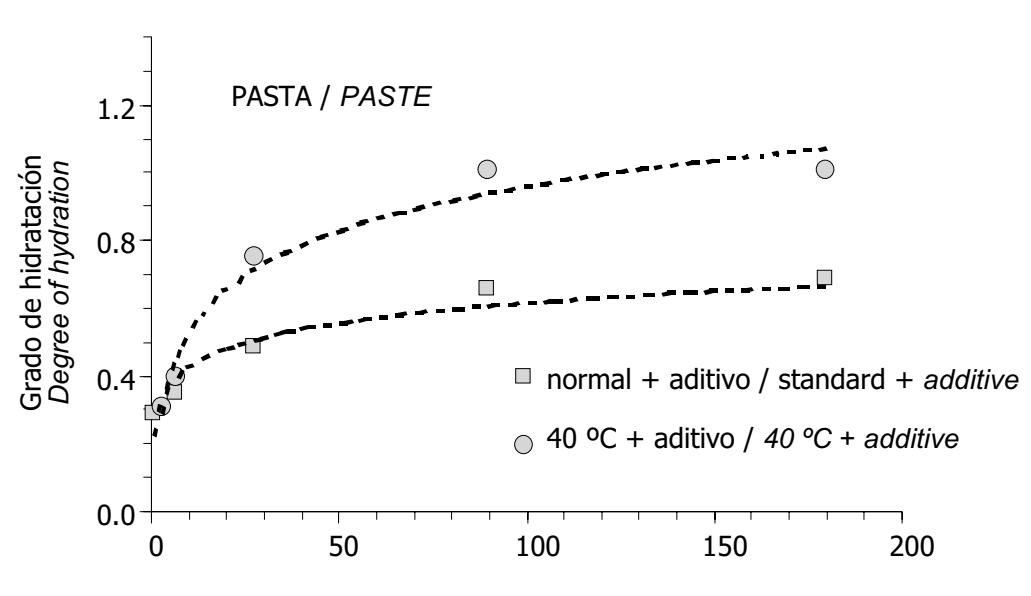

Tiempo de hidratación (días) / Hydration time (days)

Figura 4. Avance del grado de hidratación de la pasta CBCV-2-A. Influencia de las condiciones de curado. Figure 4. Degree of hydration of FABC-2-W paste. Effect of curing conditions. 

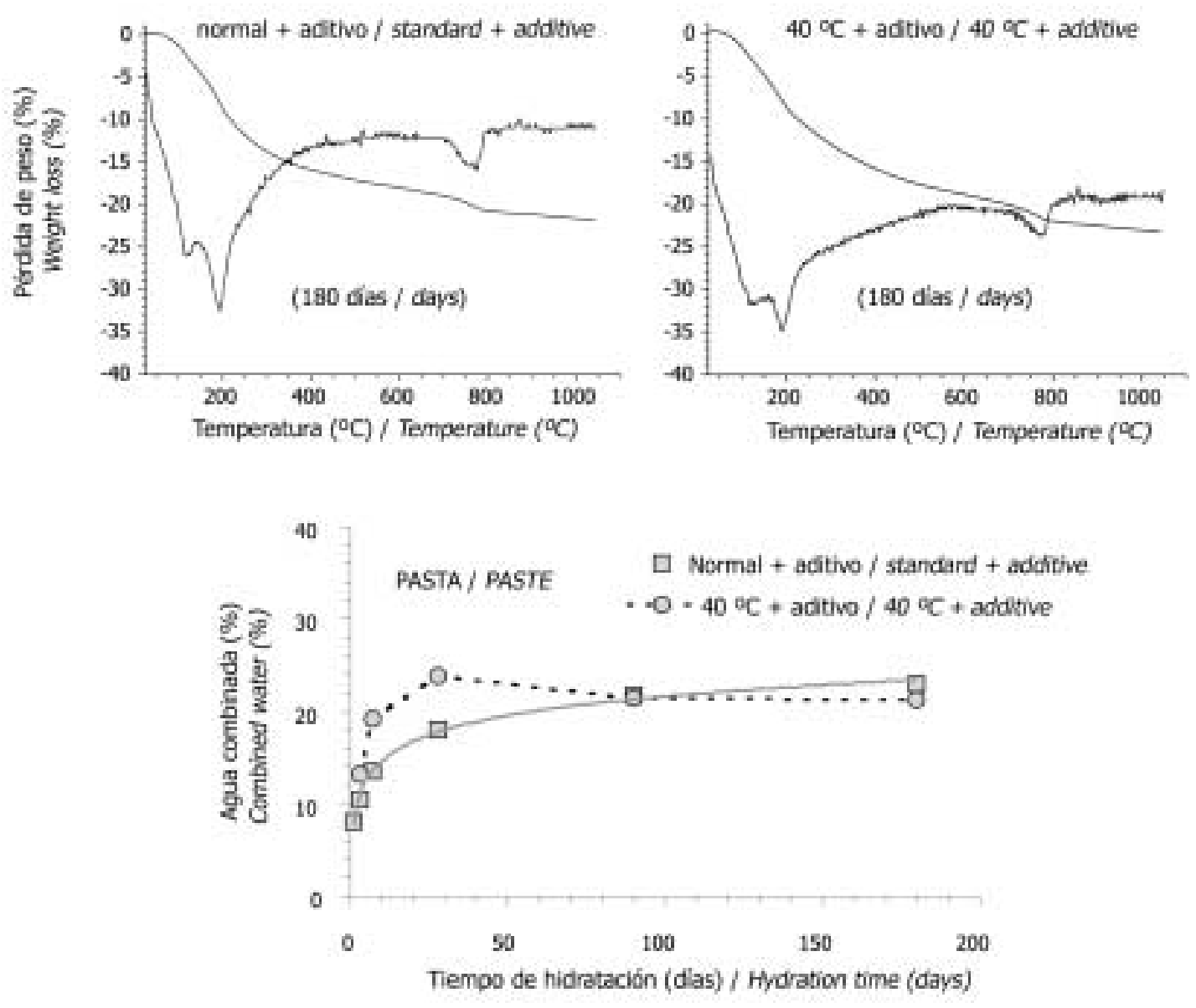

Figura 5. Curvas de TGA y DTG de las pastas de cemento CBCV-2-A hidratadas durante 180 días y evolución de agua combinada con el tiempo.

Figure 5. TGA and DTG curves for FABC-2-W pastes hydrated for 180 days and combined water content up to $t=180$ days.

Esta reacción de conversión está en total acuerdo con los resultados obtenidos por difracción de rayos $X$ (Figura 3), donde se observa la desaparición de portlandita y stratlingita y la formación de katoita.

\subsection{Resistencias mecánicas}

La resistencia mecánica a compresión de las probetas de mortero fabricadas con el cemento CBCV-2-A, así como la influencia de las condiciones de curado y la adición del aditivo superplastificante, se presentan en la Figura 6. Cada valor representa la media de 4 medidas, y las barras representan la desviación estándar de los valores.

Como era de esperar, el aditivo reductor de la demanda de agua produce un aumento de la resistencia a compresión, principalmente a primeras edades, cuando el curado es normal. En el caso del curado a $40{ }^{\circ} \mathrm{C}$, dicha
The disappearance of stratlingite as well as portlandite and the formation of katoite are consistent with the XRD results shown in Figure 3.

\subsection{Mechanical strength}

The compressive strength of FABC-2-W mortars and the effect of both curing conditions and the water-reducing additive are given in Figure 6. The values shown are the average of four measurements, with the bars depicting standard deviation.

The water-reducing additive enhanced compressive strength, particularly in early age mortars cured under standard conditions. Seven-day compressive strength was substantially higher in specimens cured at $40^{\circ} \mathrm{C}$ 
resistencia aumenta significativamente después de 7 días (desde $7 \mathrm{MPa}$ a $19 \mathrm{MPa}$, para un curado normal y a $40{ }^{\circ} \mathrm{C}$, respectivamente), pero disminuye después de 90 días hasta un valor de $15 \mathrm{MPa}$.

Este comportamiento es similar al observado en el caso del agua combinada, donde hay una disminución con el tiempo cuando el curado se ha realizado a $40{ }^{\circ} \mathrm{C}$. Si se produce la conversión de fases hidratadas hexagonales tales como la stratlingita $\left(\mathrm{Ca}_{2} \mathrm{Al}_{2} \mathrm{SiO}_{7} \cdot 8 \mathrm{H}_{2} \mathrm{O}\right)$ en fases cúbicas como la katoita $\left[\mathrm{Ca}_{3} \mathrm{Al}_{2}\left(\mathrm{SiO}_{4}\right)(\mathrm{OH})_{8}\right]$, la liberación de moléculas de agua junto con el cambio de morfología de los cristales (de hexagonal a cúbico) podría producir un aumento en la porosidad y consecuentemente una caída en los valores de resistencia a compresión. Esta hipótesis será confirmada por los análisis de porosidad de la Figura 8.

En la Figura 7 los valores de resistencia a compresión de probetas de mortero a los 28 días se comparan con valores previos publicados para CBCV-1-A (33). Como se puede apreciar, el CBCV-2-A tiene valores de resistencia 2,8 veces superiores a los del CBCV-1-A, en el caso del curado normal; y 1,3 veces superiores para el curado a $40{ }^{\circ} \mathrm{C}+$ aditivo. La alta demanda de agua del CBCV-1 (1,25 frente a 1 y 1,05 frente 0,85 para curado normal sin aditivo y con aditivo, respectivamente) junto con la menor reactividad de la ceniza volante podría ser, entre otras, la razón para explicar su baja resistencia a compresión.

\subsection{Porosidad y distribución del tamaño de poro}

Las curvas de distribución del tamaño de poro de las probetas de mortero fabricadas con el cemento CBCV-2-A y el porcentaje de poros con un diámetro medio de $>1 \mu \mathrm{m}$;
(19 compared to $7 \mathrm{MPa}$ for standard curing), but decreased after 90 days to $15 \mathrm{MPa}$.

Similar behaviour was observed for combined water, which decreased over time in mortars cured at $40{ }^{\circ} \mathrm{C}$. The conversion of a hydrated hexagonal compound such as stratlingite $\left(\mathrm{Ca}_{2} \mathrm{Al}_{2} \mathrm{SiO}_{7} .8 \mathrm{H}_{2} \mathrm{O}\right)$ into cubic katoite $\left[\mathrm{Ca}_{3} \mathrm{Al} \mathrm{I}_{2}\right.$ $\left.\left(\mathrm{SiO}_{4}\right)(\mathrm{OH})_{8}\right]$ and the concomitant release of water may increase porosity and consequently reduce compressive strength; this was confirmed by the results of the porosity analysis shown in Figure 8, discussed below.

FABC-2-W mortar 28-day compressive strength is compared to values previously published for $F A B C-1-W$ (33) mortars in Figure 7. Under standard curing conditions, compressive strength was 2.8 times higher in FABC-2-W than in FABC-1-W specimens and 1.3 times higher when they were cured at $40{ }^{\circ} \mathrm{C}$ and contained the additive. The higher the water uptake in FABC-1 (1.25 vs 1 and 1.05 vs 0.85 for standard curing conditions without and with the additive, respectively) and the lower reactivity of the fly ash may be, among others, the reasons for such lower compressive strength.

\subsection{Porosity and pore size distribution}

The pore size distribution curves for FABC-2-W mortar specimens and the percentage of pores in the diameter intervals $>1 \mu \mathrm{m} ; 0.1-1 \mu \mathrm{m}$ and $<0.1 \mu \mathrm{m}$ are given in

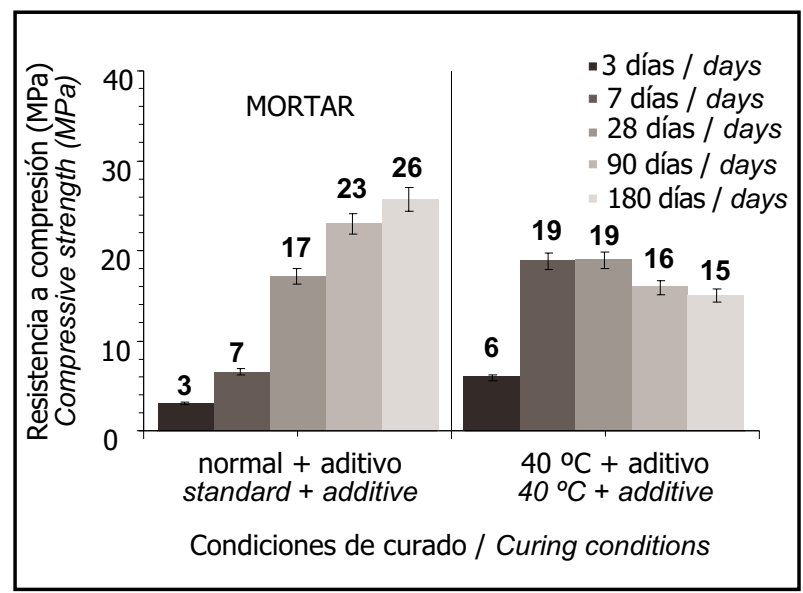

Figura 6. Influencia de las condiciones de curado y del aditivo en las resistencias mecánicas a compresión de probetas de mortero CBCV-2-A.

Figure 6. Effect of curing conditions and additive on the compressive strength of FABC-2-W mortars.

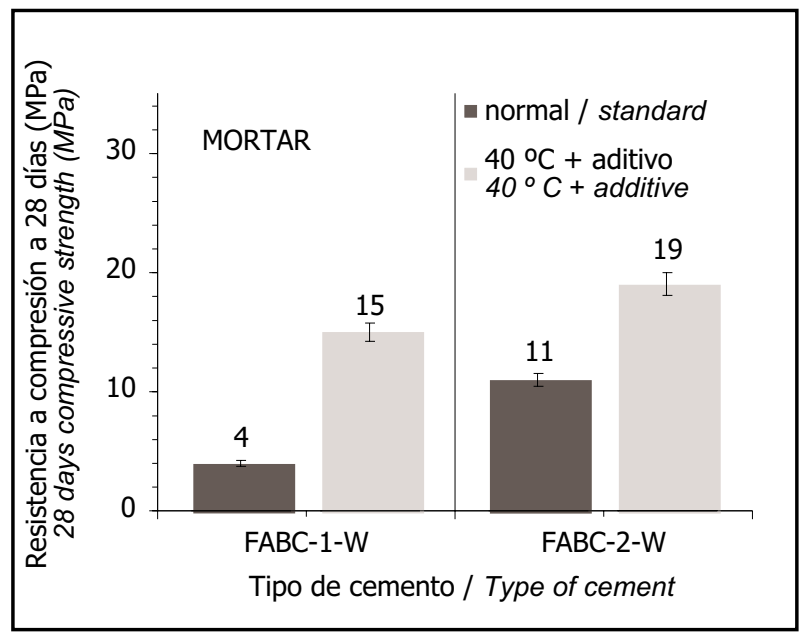

Figura 7. Influencia del tipo de cemento CBCV en las resistencias mecánicas a compresión a los 28 días.

Figure 7. Effect of FABC-type cement on 28-days compressive strength. 
$0,1-1 \mu \mathrm{m}$ y $<0,1 \mu \mathrm{m}$ se presentan en las Figuras 8 y 9 . Cubos de alrededor de $1 \mathrm{~cm}^{3}$ se desgasificaron previamente a vacío a temperatura ambiente.

Como se aprecia en la Figura 8, en el caso del curado normal sin aditivo, la porosidad total disminuye con el tiempo desde $22 \%$ después de 7 días a $16 \%$ (en peso) después de 180 días; cambios más relevantes se producen en el diámetro medio de poro, el cual se desplaza a valores menores. En particular, los poros comprendidos en el rango de 0,1-1 $\mu \mathrm{m}$ (Figura 9) disminuyen desde un $12,8 \%$ (en peso) después de 7 días de hidratación al $1,0 \%$ después de 180 días; y los poros más pequeños, comprendidos en el rango de $<0,1 \mu \mathrm{m}$ aumentan desde un $6,9 \%$ después de 7 días a un $14,4 \%$ después de 180 días, debido a la formación del gel C-S-H.
Figures 8 and 9. Cubic specimens measuring about $1 \mathrm{~cm}^{3}$ were degasified in a vacuum at room temperature prior to testing.

As Figure 8 shows, in specimens cured under standard conditions without the additive, total porosity declined from $22 \%$ after 7 days to $16 \%$ (by weight) after 180 days; more relevant changes were observed in the mean pore-size diameter, which shifted to substantially lower values in the same period of time. More specifically, pores in the 0.1-1 $\mu \mathrm{m}$ range (Figure 9) accounted for $12.8 \%$ (by weight) of total porosity after seven days of hydration, but only for $1.0 \%$ after 180 days; the proportion of pores in the $<0.1 \mu \mathrm{m}$ range, in turn, rose from $6.9 \%$ after seven days to $14.4 \%$ after 180 days.

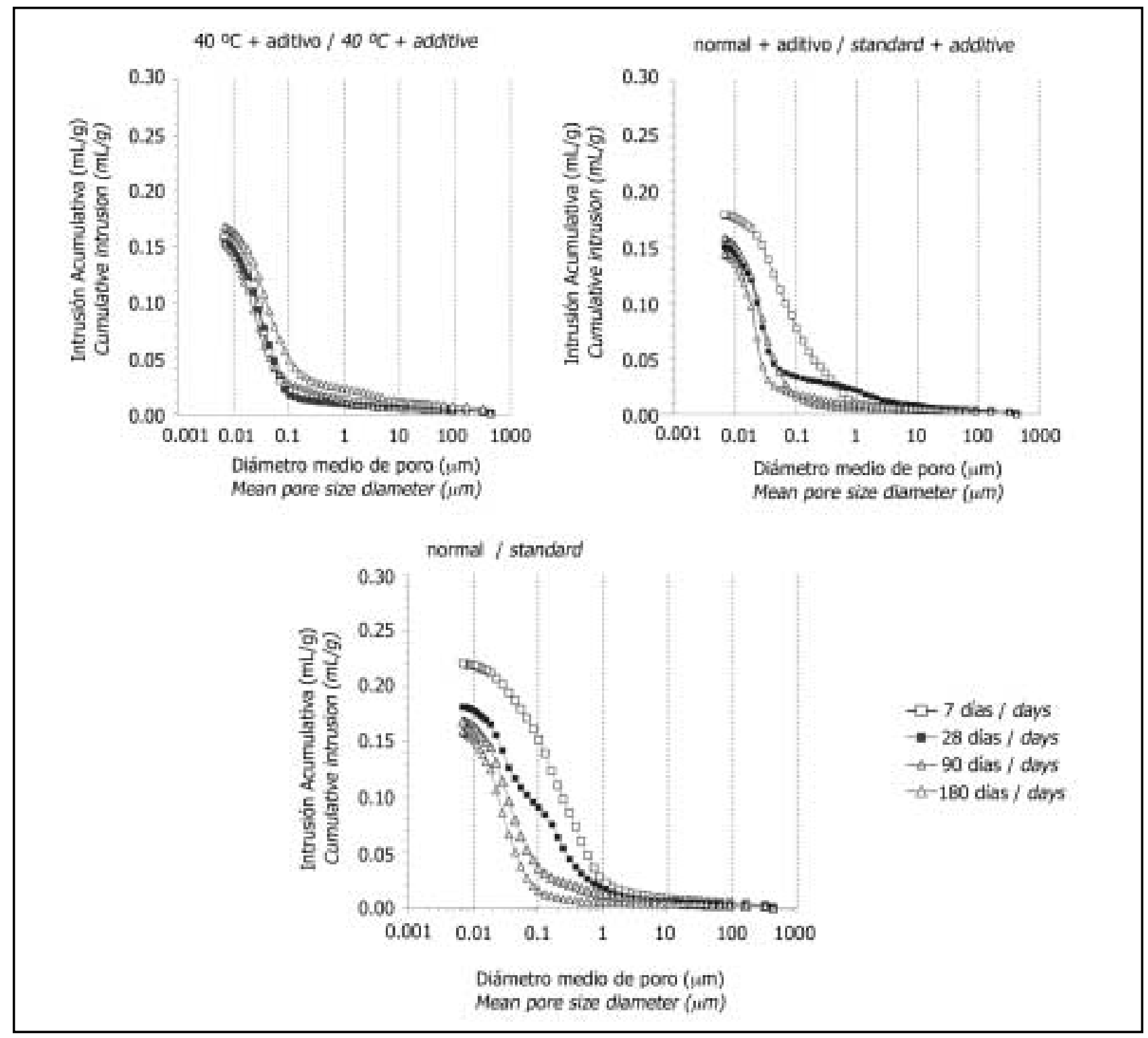

Figura 8. Influencia de las condiciones de curado en la distribución del tamaño de poro del mortero CBCV-2-A. Figure 8. Effect of curing conditions on pore size distribution in FABC-2-W mortar specimens. 
La presencia del aditivo hace disminuir tanto la porosidad total como el diámetro medio de poro, aun después de 7 días de hidratación. Así, los poros comprendidos en el rango de 0,1-1 $\mu \mathrm{m}$ (Figura 9) disminuyen desde un 5,8\% (en peso) después de 7 días de hidratación al 0,7\% después de 180 días; se produce menos influencia en los poros finos comprendidos en el rango de $<0,1 \mu \mathrm{m}$, los cuales aumentan desde un $11,4 \%$ después de 7 días hasta un $13,1 \%$ después de 180 días.

Cuando se realiza el curado a $40^{\circ} \mathrm{C}$ los valores de porosidad total prácticamente no presentan diferencias entre 7 y 180 días, pero hay un aumento importante en la cantidad de poros grandes desde 28 hasta 180 días. Así, los poros comprendidos en rango 0,1-1 $\mu \mathrm{m}$ (Figura 9) aumentan desde $0,9 \%$ (en peso) después de 28 días de hidratación hasta un 2,6\% después de 180 días; y los poros comprendidos en el rango $>1 \mu \mathrm{m}$ aumentan desde un $0,8 \%$ a los 7 días hasta 2,2\% después de 180 días. El aumento de poros grandes se produce por la disminución de los poros finos, donde se pasa del $13,8 \%$ al $12 \%$, hecho que está relacionado con la hipótesis anteriormente comentada de la conversión de productos hidratados, causada por la temperatura de curado, así como con la bajada en resistencias a compresión de la Figura 6 y la caída del contenido en agua combinada (Figura 5).
The additive lowered both total porosity and the mean pore size diameter even after just seven days of hydration; moreover, the proportion of pores in the 0.1-1 $\mu \mathrm{m}$ range (Figure 9) fell from $5.8 \%$ (by weight) after seven days of hydration to $0.7 \%$ after 180 days. The effect on pores in the $<0.1 \mu \mathrm{m}$ range was smaller, with the proportion rising from $11.4 \%$ after seven days to $13.1 \%$ after 180.

While specimens cured at $40{ }^{\circ} \mathrm{C}$ exhibited practically no difference in seven- and 180-day total porosity, a significant increase in the amount of large pores was recorded between 28 and 180 days. The percentage of pores in the 0.1-1 $\mu \mathrm{m}$ range (Figure 9) climbed from $0.9 \%$ (by weight) after 28 days of hydration to $2.6 \%$ after 180 days, while the percentage in the $>1 \mu \mathrm{m}$ range grew from $0.8 \%$ after seven days to $2.2 \%$ after 180 days. The increase in the proportion of large pores took place at the expense of the percentage of fine pores, which dipped from $13.8 \%$ to $12 \%$. This is consistent with the above hypothesis to the effect that the curing temperature is behind the conversion of hydrated products, the decline in compressive strength depicted in Figure 6 and the lower combined water content shown in Figure 5.
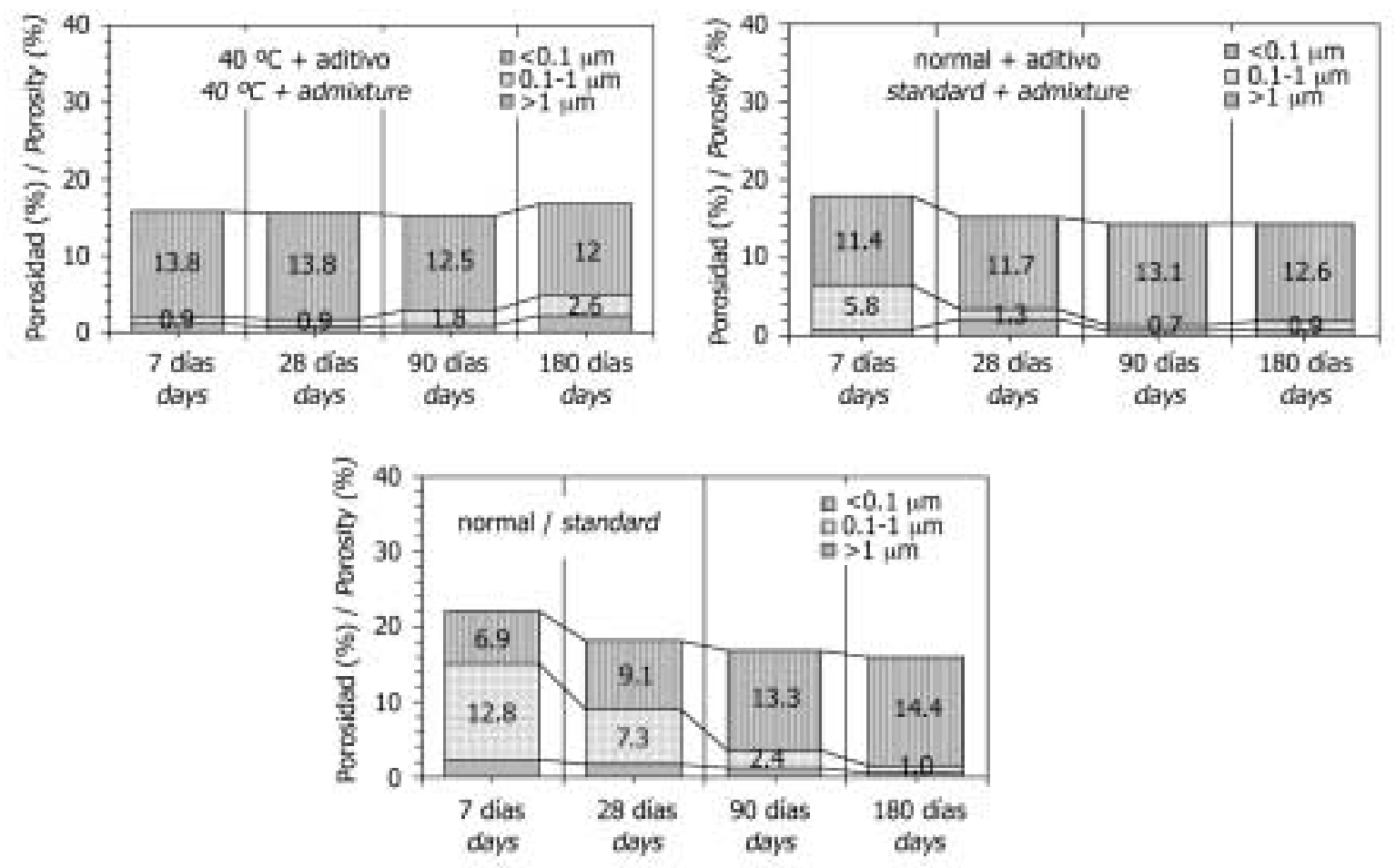

Figura 9. Influencia de las condiciones de curado en el diferente tamaño de poro de las probetas de mortero CBCV-2-A. Figure 9. Effect of curing conditions on different pore size intervals in FABC-2-W mortar specimens. 


\subsection{Correlaciones cuantitativas}

Para finalizar, en la Figura 10 se presentan las correlaciones cuantitativas obtenidas entre los diferentes parámetros estudiados. En el caso del curado normal existe una buena correlación lineal si se cruzan los valores de resistencias mecánicas a compresión con el grado de hidratación y el agua combinada. En el caso del grado de hidratación, es necesario un valor mínimo del mismo de 0,20 para que el cemento desarrolle suficientes resistencias que puedan ser medidas y corroboradas. Este valor está en consonancia con los presentados en la Figura 7 de la referencia 37. Los tres puntos que están fuera de la regresión lineal corresponden a las probetas curadas a largas edades y a $40{ }^{\circ} \mathrm{C}$, que presentan valores de resistencia mecánica menores debido al proceso de conversión mencionado anteriormente.

La correlación lineal obtenida entre la resistencia mecánica a compresión y el contenido de agua combinada indica un valor mínimo de agua del $9 \%$, necesaria para que el cemento comience a desarrollar resistencia. Hecho idéntico al comentado en el párrafo anterior ocurre con los tres puntos de las probetas curadas a $40^{\circ} \mathrm{C}$.

Se obtiene una relación lineal entre el grado de hidratación, calculado a partir de los datos de DRX, y los

\subsection{Quantitative correlations}

Finally, the correlations among the various parameters studied are shown in Figure 10. Good linear relationships are obtained when the compressive strength values are plotted against degree of hydration and combined water in specimens cured under standard conditions. A minimum value for the degree of hydration of approximately 0.20 must be reached for the cement to develop recordable strength during testing. This value concurs with a finding reported earlier (Figure 7, ref. 37). The three points outside the linear regression correspond to later age samples cured at $40{ }^{\circ} \mathrm{C}$ whose mechanical strength values are lower due to the conversion process described above.

According to the linear correlation found for compressive strength and combined water content, a minimum of approximately $9 \%$ of bound water is needed for the cement to start to develop strength. The three points corresponding to specimens cured at $40^{\circ} \mathrm{C}$ also exhibited lower compressive strength due to conversion of the initial minerals to katoite.

A correlation was found for the degree of hydration as determined by XRD analysis and the combined water
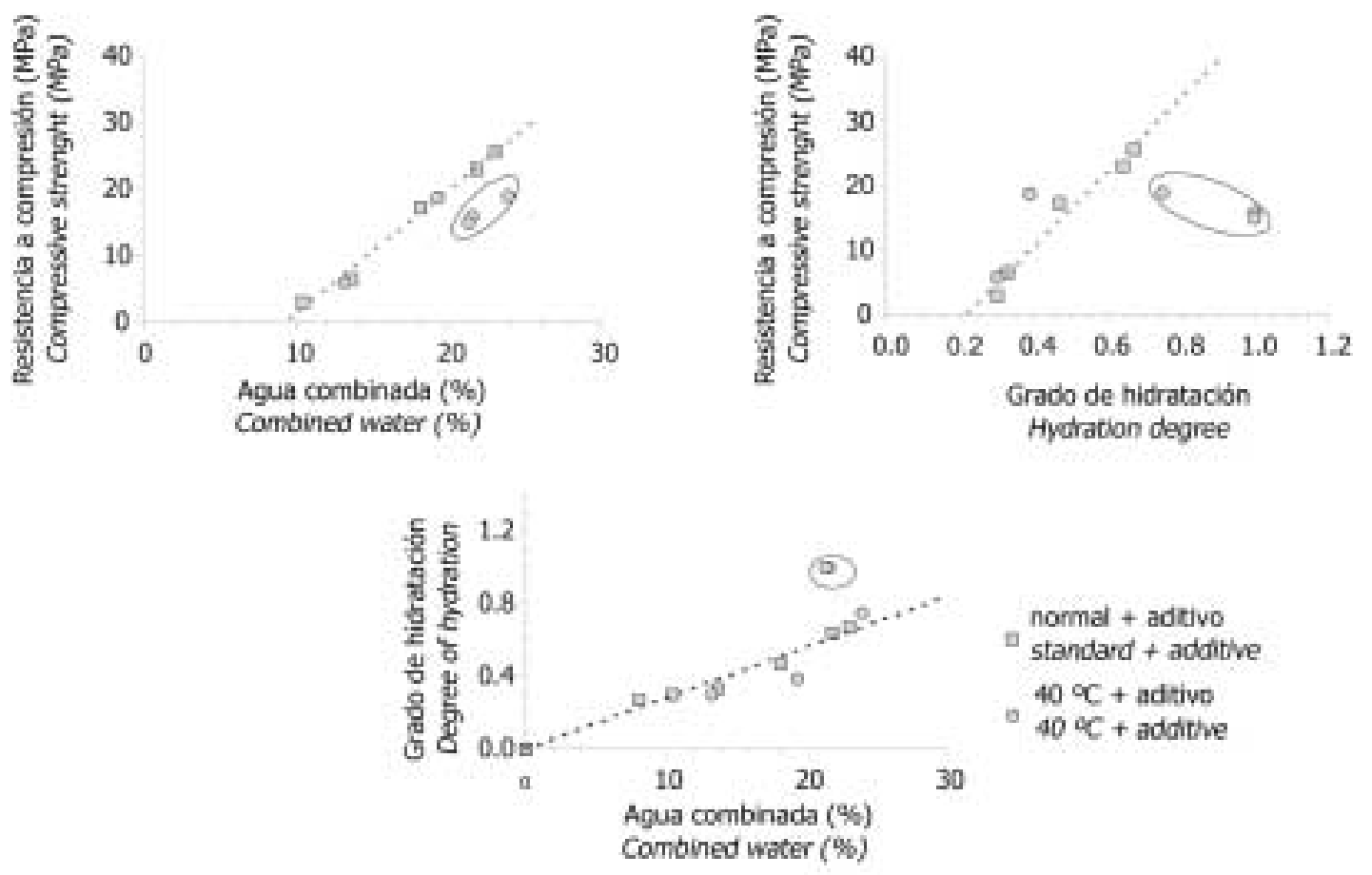

Figura 10. Correlaciones cuantitativas entre diferentes parámetros.

Figure 10. Correlations among different parameters. 
valores del agua combinada. Los puntos marcados, situados fuera de la correlación lineal, corresponden a las pastas curadas a $40{ }^{\circ} \mathrm{C}$ a largas edades donde se formaba katoita, por lo que el contenido de agua combinada es menor.

\section{CONCLUSIONES GENERALES}

- El aditivo reductor de la demanda de agua aumenta las resistencias mecánicas a compresión, principalmente a edades tempranas de hidratación ( $40 \%$ a 7 días) para un curado a temperatura ambiente (curado normal). En el caso del curado a $40{ }^{\circ} \mathrm{C}$, las resistencias mecánicas aumentan significativamente después de 7 días (desde $7 \mathrm{MPa}$ a $19 \mathrm{MPa}$, para curado a temperatura normal y a $40{ }^{\circ} \mathrm{C}$, respectivamente), pero decaen después de 90 días a valores de $15 \mathrm{MPa}$.

- La bajada de resistencias es causada por la formación de la fase cúbica katoita $\left[\mathrm{Ca}_{3} \mathrm{Al}_{2}\left(\mathrm{SiO}_{4}\right)(\mathrm{OH})_{8}\right]$ procedente de la hexagonal stratlingita $\left(\mathrm{Ca}_{2} \mathrm{Al}_{2} \mathrm{SiO}_{7} \cdot 8 \mathrm{H}_{2} \mathrm{O}\right)$. Esta conversión produce la liberación de moléculas de agua y cambios en la morfología de los cristales (de hexagonal a cúbico). Por ello, la distribución del tamaño de poro produce un aumento de los poros mayores $(>1 \mu \mathrm{m})$ y una disminución de los poros más finos $(<0,1 \mu \mathrm{m})$.

- Los resultados de agua combinada corroboran la conversión antes mencionada, que causa una bajada en las resistencias mecánicas.

- Las principales fases hidratadas formadas a una temperatura normal de curado son: $\mathrm{C}_{2} \mathrm{SH}_{0,35}$, monosulfoaluminato de calcio hidratado $\left[\mathrm{Ca}_{4} \mathrm{Al}_{2}\left(\mathrm{SO}_{4}\right) \mathrm{O}_{6} \cdot 10 \mathrm{H}_{2} \mathrm{O}\right]$ y stratlingita $\left(\mathrm{Ca}_{2} \mathrm{Al}_{2} \mathrm{SiO}_{7} \cdot 8 \mathrm{H}_{2} \mathrm{O}\right)$ ambas tipo $\mathrm{AFm}$, gel C-S-H $\left(\mathrm{Ca}_{1,5} \mathrm{SiO}_{3,5} \cdot \mathrm{H}_{2} \mathrm{O}\right)$ y trazas de portlandita $\left[\mathrm{Ca}(\mathrm{OH})_{2}\right]$.

- Con el curado a $40{ }^{\circ} \mathrm{C}$, las principales diferencias encontradas fueron: la formación de katoita $\left[\mathrm{Ca}_{3} \mathrm{Al}_{2}\left(\mathrm{SiO}_{4}\right)(\mathrm{OH})_{8}\right]$ a largas edades y la desaparición de portlandita; la ausencia de stratlingita; la formación de monocarbo-aluminato de calcio hidratado $\left[\mathrm{Ca}_{4} \mathrm{Al}_{2} \mathrm{O}_{6}\left(\mathrm{CO}_{3}\right) \cdot 11 \mathrm{H}_{2} \mathrm{O}\right]$, quizás debido a la carbonatación del $\mathrm{Ca}_{4} \mathrm{Al}_{2}\left(\mathrm{SO}_{4}\right) \mathrm{O}_{6} \cdot 10 \mathrm{H}_{2} \mathrm{O}$ y la disminución del $\mathrm{C}_{2} \mathrm{SH}_{0,35}$.

- En el caso del curado normal se obtiene una buena correlación entre los diferentes parámetros estudiados. La representación de la resistencia a compresión frente al grado de avance de la hidratación indica que, como mínimo, necesitamos un grado de hidratación de 0,20 para alcanzar resistencias. Del mismo modo, la gráfica de resistencia mecánica a compresión frente al contenido de agua combinada indica que, como mínimo, se necesita un 9\% de agua combinada para alcanzar valores significativos de resistencias. content. The circled points located off the correlation line correspond to older specimens cured at $40^{\circ} \mathrm{C}$, in which katoite had formed and the bound water content was therefore lower.

\section{GENERAL CONCLUSIONS}

- The water-reducing additive increased compressive strength (by $40 \%$ after seven days), particularly in early age specimens cured under standard conditions. Curing at $40^{\circ} \mathrm{C}$ raised seven-day compressive strength substantially (19 compared to $7 \mathrm{MPa}$ for standard curing), although strength dropped to $15 \mathrm{MPa}$ after 90 days.

- The decline in compressive strength is due to the formation of cubic katoite $\left[\mathrm{Ca}_{3} \mathrm{Al}_{2}\left(\mathrm{SiO}_{4}\right)(\mathrm{OH}) \mathrm{SiO}_{48}\right]$ at the expense of hexagonal stratlingite $\left(\mathrm{Ca}_{2} \mathrm{Al}_{2} \mathrm{SiO}_{7} 8 \mathrm{H}_{2} \mathrm{O}\right)$ and the concomitant release of water molecules. Pore size distribution was affected as a result, with an increase in the proportion of large pores $(>1 \mu \mathrm{m})$ and a decrease in the percentage of fine pores $(<0.1 \mu \mathrm{m})$.

- The findings for combined water content corroborated the aforementioned conversion, which caused strength to drop.

- The hydrated phases forming under standard conditions were: $\mathrm{C}_{2} \mathrm{SH}_{0.35}$, AFm-type hydrated calcium-monosulpho-aluminate $\left[\mathrm{Ca}_{4} \mathrm{Al}_{2}\left(\mathrm{SO}_{4}\right) \mathrm{O}_{6} \cdot 1 \mathrm{H}_{2} \mathrm{O}\right]$ and stratlingite $\left(\mathrm{Ca}_{2} \mathrm{Al}_{2} \mathrm{SiO}_{7} 8 \mathrm{H}_{2} \mathrm{O}\right), \mathrm{C}-\mathrm{S}-\mathrm{H}$ gel $\left(\mathrm{Ca}_{1.5} \mathrm{SiO}_{3.5} \times \mathrm{H}_{2} \mathrm{O}\right)$ and traces of portlandite $\left[\mathrm{Ca}(\mathrm{OH})_{2}\right]$.

- The chief differences found in specimens cured at $40{ }^{\circ} \mathrm{C}$ were: katoite $\left[\mathrm{Ca}_{3} \mathrm{Al}{ }_{2}\left(\mathrm{SiO}_{4}\right)(\mathrm{OH})_{8}\right]$ formation and the disappearance of portlandite at older ages; the absence of stratlingite; the formation of hydrated calciummonocarbo-aluminate $\left[\mathrm{Ca}_{4} \mathrm{Al}_{2} \mathrm{O}_{6}\left(\mathrm{CO}_{3}\right) \cdot 11 \mathrm{H}_{2} \mathrm{O}\right]$, perhaps as a result of $\mathrm{Ca}_{4} \mathrm{Al}_{2}\left(\mathrm{SO}_{4}\right) \mathrm{O}_{6} \cdot 10 \mathrm{H}_{2} \mathrm{O}$ carbonation and the decline in $\mathrm{C}_{2} \mathrm{SH}_{0.35}$ content.

- A good correlation was obtained for the various parameters studied. The graph depicting the relationship between compressive strength and the degree of hydration shows that strength did not develop until the degree of hydration reached approximately 0.20. Similarly, the compressive strength versus combined water content graph shows that no significant compressive strength develops at water content values of under $9 \%$. 


\section{AGRADECIMIENTOS}

Los autores agradecen la financiación por parte del Ministerio de Ciencia y Tecnología mediante la concesión del proyecto (MAT 2002-04023-CO3) para la realización de estos trabajos; así como a la Central Térmica de Cers (Cataluña) por el suministro de la ceniza volante.

\section{ACKNOWLEDGEMENTS}

This research was funded by the Ministry of Science and Technology under project number MAT 2002-04023CO3. The authors wish to thank the Cercs steam power plant in Catalonia for providing the fly ash used in this study.

\section{BIBLIOGRAFÍA/BIBLIOGRAPHY}

(1) http://www.ecoba.com/index.html.

(2) Intergovernmental Panel on Climate Change: Special report on Emissions 2001. http://www.grida.no/climate.

(3) McCaffrey, R.: "Climate change and the cement industry", Global Cement and Lime Magazine: Environmental Special Issue, 2002.

(4) European Cement Industry: Cembureau (Organisation of the Cement Industry in Europe). http://www.cembureau.be.

(5) Gartner, E.: "Industrially Interesting Approaches to "low-CO ${ }_{2}$ " Cements", Cem. Concr. Res., vol. 34 (2004), pp. 1489-1498.

(6) Popescu, C. D., Muntean, M. y Sharp, J. H.: "Industrial Trial Production of Low Energy Belite Cement", Cem. \& Concr. Comp., vol. 25 (2003), pp. 689-693.

(7) Guo, S-H., Zhang, H-T., Zhen, L., Zhang, W-S. y Chen, Y-M.: "The Formation and Performance of High Belite Cement Clinker with Different Interstitial Phase Content", Proceedings of the $11^{\text {th }}$ International Congress on the Chemistry of Cement (ICCC) "Cement's Contribution to the Development in the 21 st Century", edited by G. Grieve and G. Owens. Durban, South Africa, 2003, pp. $1027-1034$.

(8) Singh, Nakshatra B., Rai, Sarita y Singh, Neelam: "Highly Reactive b-Dicalcium Silicate", J. Am. Ceram. Soc., vol. 85, no 9 (2002), pp. 2171-2176.

(9) Fukuda, Koichiro, Wakamatsu, Norihiko y Ito, Suketoshi: "Improvement in Hydration Reactivity of a-phase Belite by Remelting Reaction", J. Am. Ceram. Soc., vol. 84, no 3 (2001), pp. 639-641.

(10) Fukuda, K. y Taguchi, H.: "Hydration of $a^{\prime}{ }^{\prime}$-and b-dicalcium Silicates with Identical Concentration of Phosphorus Oxide", Cem. Concr. Res., vol. 29 (1999), pp. 503-506.

(11) Hong, S. H. y Young, J. F.: "Hydration Kinetics and Phase Stability of Dicalcium Silicate Synthesized by the Pechini Process", J. Amer. Ceram. Soc., vol. 82, no 7 (1999), pp. 1681-1686.

(12) Chatterjee, A. K.: "High Belite Cements-Present Status and Future Technological Options: Part I", Cem. Concr. Res., vol. 26, no 8 (1996), pp. 1213-1225.

(13) Chatterjee, A. K.: "Future Technological Options: Part II", Cem. Concr. Res., vol. 26, no 8 (1996), pp. 1227-1237.

(14) Young, J. F.: "Highly Reactive Dicalcium Silicates for Belite Cement", Proceedings of the RILEM International Conference "Concrete: from Material to Structure, pp. 1-15. Edited by J. P. Bournazel and Y. Malier. Arlés, France, 1996.

(15) Moranville-Regourd, M. y Boikova, A.: "Chemistry, Structure, Properties and Quality of Clinker", Proceedings of the 9th International Congress on the Chemistry of Cement, New Delhi, edited by A.K. Mullick. National Council for Cement and Buildings Materials. New Delhi (1992), pp. 3-48.

(16) Ishida, H., Mabuchi, K., Sasaki, K. y Mitsuda, T.: "Low Temperature Synthesis of b-Ca ${ }_{2} \mathrm{SiO}_{4}$ from Hillebrandite", J. Amer. Ceram. Soc., vol. 75, no 9 (1992), pp. 2427-2432.

(17) Gies, A. y Knöfel, D.: "Influence of Alkalis on the Composition of Belite-Rich Cement Clinkers and the Technological Properties of the Resulting Cements", Cem. Concr. Res., vol. 16 (1986), pp. 411-422.

(18) Suzuki, K.: "Hydration and Strength of a-, a'- and b- Dicalcium Silicates Stabilized with Na-Al, K-Al, Na-Fe and K-Fe", Proceedings of the 7th International Congress of the Chemistry of Cement, pp. II-47 (Editions Septima), París, 1980.

(19) Roy, D. M. y Oyefesobi, S. O.: "Preparation of Very Reactive $\mathrm{Ca}_{2} \mathrm{SiO}_{4}$ Powder", J. Amer. Ceram. Soc., vol. 60 (1977), pp. 178-180.

(20) Ishida, H. y Mitsuda, T.: "Science and Technology of Highly Reactive b-Dicalcium Silicate: Preparation, Hydration and Gels", Materials Science of Concrete V, edited by J. Skalny and S. Mindess. The American Ceramic Society, Westerville, Ohio (1998), pp. 1-44.

(21) Bonafous, L., Bessada, C., Massiot, D., Coutures, J-P., Rolland, B. y Colombet, P.: "Si MAS NMR Study of Dicalciumsilicate - The Structural Influence of Sulfate and Alumina Stabilisers", J. Am. Ceram. Soc., vol. 78, no 10 (1995), pp. 2603-2608.

(22) Jiang, W. y Roy, D. M.: "Hydrothermal Processing of New Fly Ash Cement", Ceram. Bull., vol. 71, no 4 (1992), pp. $642-647$.

(23) Guerrero, A., Goñi, S., Macías, A. y Luxán, M. P.: "Effect of the Starting Fly Ash on the Microstructure and Mechanical Properties of Fly Ash-Belite Cement Mortars", Cem. Concr. Res., vol. 30, no 4 (2000), pp. 553-559.

(24) Goñi, S., Guerrero, A., Luxán, M. P. y Macías, A.: "Dehydration of Pozzolanic Products Hydrothermally Synthesized from Fly Ashes. Microstructure Evolution", Mater. Res. Bull. vol. 35, no 8 (2000), pp. 1333-1344.

(25) Guerrero, A., Goñi, S., Macías, A. y Luxán, M. P.: "Hydraulic Activity and Microstructural Characterization of New Fly Ash-Belite Cements Synthesized at Different Temperatures", J. Mater. Res., vol. 14, no 6 (1999), pp. 2680-2687. 
(26) Guerrero, A., Goñi, S., Macías, A. y Luxán, M. P.: "Mechanical Properties, Pore-Size Distribution and Pore Solution of Fly Ash-Belite Cement Mortars", Cem. Concr. Res., vol. 29 (1999), pp. 1753-1758.

(27) Guerrero, A., Goñi, S. y Macías, A.: "Durability of new Fly ash-Belite Cement Mortars in Sulfated and Chloride médium", Cem. Concr. Res., vol. 30, no 8 (2000), pp. 1231-1238.

(28) Guerrero, A., Goñi, S., Macías, A. y Fernández, E.: "Microstructure of New Fly Ash-Belite Cement Mortar: Changes Provoked by Sulphate, Chloride and Sodium Ions", Proceedings of $21^{\text {th }}$ International Conference on Cement Microscopy, edited by L. Jany y A. Nisperos. Published by the International Cement Microscopy Association, Las Vegas (Nevada) (1999), pp. 230-237.

(29) Goñi, S., Guerrero, A., Luxán, M. P. y Macías, A.: "Activation of the Fly Ash Pozzolanic Reaction by Hydrothermal Conditions", Cem. Concr. Res., vol. 33 (2003), pp. 1399-1405.

(30) Guerrero, A., Goñi, S., Campillo, I. y Moragues, A.: "Belite Cement Clinker from Coal Fly Ash of High Ca Content. Optimization of Synthesis Parameters". Environmental Science and Technology, vol. 38, no 11 (2004), pp. 3209-3213.

(31) Goñi, S., Elizalde, Guerrero, A., Bustos, Moragues, A., Terradas, Tallafigo, M. F., Vidal, Campillo, I., Santos, Sánchez, J., Dolado y Porro, A., Fernández: New Belite Cement Clinkers from Fly ash of Coal Combustion of High Ca Content. Spanish Patent No ES2199059, published 1, February, 2005.

(32) Goñi, S., Guerrero, A., Lorenzo, M. P., Moragues, A., Campillo, I. y Dolado, J. S.: High Calcium Coal Fly Ash as Raw Material for Synthesizing New Low-Energy Cements. In Proceedings of Eighth CANMET/ACI International Conference on Fly Ash, Silica Fume, Slag and Natural Pozzolans in Concrete, edited by V. M. Malhotra, Michigan (2004), pp. 381-394.

(33) Guerrero, A., Goñi, S., Moragues, A. y Dolado, J. S.: "Microstructure and Mechanical Performance of Belite Cements from High Calcium Coal Fly Ash", J. Am. Ceram. Soc., vol. 88, no 7 (2005), pp. 1845-1853.

(34) Goñi, S., Guerrero, A. y Lorenzo, M. P.: "Microscopy Study of Belite Phases Obtained from Coal Fly Ash Class C: Influence of Heating", Proceedings of 10th Euroseminar on Microscopy Applied to Building Materials, Pasley, june 22-25, 2005.

(35) Guerrero, A., Goñi, S., Moragues, A. y Campillo, I.: "Changes of the BET- $\mathrm{N}_{2}$ - Specific Surface Area and Pore Volume during Hydration of Fly Ash Belite Cement", Proceedings of $2^{\text {nd }}$ International Symposium on Nanotechnology in Construction, november 13-16, 2005, Bilbao, Spain.

(36) Taylor, H. F. W., Cement Chemistry, Chapter 6. Hydrated Aluminate, Ferrite and Sulphate Phases, edited by H.F.W.Taylor Academic Press, London (1992), pp. 167-198.

(37) Johansen, V. y Idorn, G. M.: "Cement Production and Cement Quality", Materials Science of Concrete I, edited by J. Skalny, the American Ceramic Society, Inc. Westerville, OH (1989), pp. 27-71. 